\title{
Energy-Entropy-Momentum integration of discrete thermo-visco-elastic dynamics
}

\author{
Juan Carlos García Orden ${ }^{\mathrm{a}, *}$, Ignacio Romero ${ }^{\mathrm{b}}$ \\ ${ }^{a}$ ETSI Caminos, Technical University of Madrid. c/ Profesor Aranguren s/n, 28040 \\ Madrid. Spain \\ ${ }^{b}$ ETSI Industriales, Technical University of Madrid. c/ José Gutierrez Abascal 2, 28006 \\ Madrid. Spain
}

\begin{abstract}
A novel time integration scheme is presented for the numerical solution of the dynamics of discrete systems consisting of point masses and thermovisco-elastic springs. Even considering fully coupled constitutive laws for the elements, the obtained solutions strictly preserve the two laws of thermodynamics and the symmetries of the continuum evolution equations. Moreover, the unconditional control over the energy and the entropy growth have the effect of stabilizing the numerical solution, allowing the use of larger time steps than those suitable for comparable implicit algorithms. Proofs for these claims are provided in the article as well as numerical examples that illustrate the performance of the method.
\end{abstract}

Keywords:

Time integration, structure preservation, geometric integration, coupled problems, thermodynamics

\section{Introduction}

Enormous efforts have been devoted in the last decades to the development of accurate and robust time integration methods for general evolution equations, and in particular in Mechanics. Even though conserving problems

\footnotetext{
*Corresponding author

Email addresses: juancarlos.garcia@upm.es (Juan Carlos García Orden), ignacio.romero@upm.es (Ignacio Romero)
} 
have attracted most of the attention in this respect (see, for example the recent monograph Leimkuhler and Reich (2004)), general dissipative problems are obviously extremely relevant.

With regards to accuracy, even the classical texts devoted to initial value problems (e.g., Richtmyer and Morton (1967); Gear (1971)) identified and analyzed the concept of "order of accuracy". However, it was later realized that methods with low order of accuracy could provide more precise solutions that higher order methods, especially in long term simulations. Whereas the "order of accuracy" is a well defined property of a discretization scheme, the real "accuracy" is a more elusive concept that requires further analysis. In relation to this, results obtained in the last two decades have concluded that integration schemes which are capable of preserving (part of) the qualitative features of the evolution problem they are meant to approximate are often extremely accurate in long term simulations (Hairer et al., 2002). For example, the use of symplectic methods has become widespread for Hamiltonian problems (Sanz-Serna and Calvo, 1994). For this type of problems, ubiquitous in Mechanics, Energy and Momentum conserving methods (Labudde and Greenspan, 1976a,b; Simó and Tarnow, 1992; Simó et al., 1992; González, 2000) have also become widely employed.

Structure preserving algorithms, as this type of methods are known, not only are remarkably accurate but are robust too. In particular, Energy and Momentum conserving methods control the growth of the numerical solution (and its perturbations) resulting in extremely stable methods which can often employ time step sizes one order of magnitude larger than comparable implicit methods. This statement is well documented in the literature and early numerical evidence for it can be found, for example, in (Simó and Tarnow, 1992; Simó et al., 1992; Simo and Tarnow, 1994; González and Simó, 1996).

Most energy conserving methods are second-order accurate. However if higher order accuracy is required, as for example in certain wave propagation problems, conserving methods with higher orders of accuracy can be obtained using composition, as in (Tarnow and Simo (1994)), or using space-time formulations (see Groß et al. (2005)). Bounded energy growth, and high frequency algorithmic dissipation, can be simultaneously obtained by using Energy Dissipative, Momentum Conserving methods (Armero and Romero, 2001a,b; Romero and Armero, 2000; Armero and Romero, 2003). In this way, not only structural damping is represented in an acceptable way, but all the benefits of energy control and discrete conservation are preserved. This is an important extension of conserving methods and time integration 
methods that preserve momenta, control the energy growth, and possess high frequency artificial dissipation have been proposed by many authors (Kuhl and Ramm, 1996; Botasso and Borri, 1997; Bauchau and Bottasso, 1999; Kuhl and Crisfield, 1999; Bottasso et al., 2001; Borri et al., 2001).

These two properties of structure preserving methods, namely enhanced accuracy in long term simulations and robustness, have made them very popular in Computational Mechanics. Moreover, their use in Multibody System Dynamics has also increased during the last years (García Orden and Goicolea, 2000, 2005; Betsch and Uhlar, 2007), in this case motivated by their excellent performance integrating systems with constraints.

In view of the success of structure preserving methods for conservative problems there is now interest in extending them to dissipative problems. In particular, several recent works have tried to broaden the ideas of the EnergyMomentum methods to specific dissipative methods such as frictional contact (Ortiz et al., 2000), plasticity (Meng and Laursen, 2002), viscoelasticity (Groß and Betsch, 2010), etc. To the authors' knowledge, the first attempt to systematically generalize Energy-Momentum to dissipative problems has been presented in (Romero, 2009, 2010b,a). In these works, a general methodology was presented that allows the formulation of time integration methods that strictly preserve the two laws of thermodynamics, as well as the symmetries, for general thermomechanical problems. Starting from a GENERIC reformulation of the evolution equations (Öttinger, 2005), they describe a general methodology for the design of structure preserving discretization of dissipative evolution equations. Part of the formulation process is based on the ideas of the Energy-Momentum method and the resulting algorithms share with the later some of the appealing features discussed above which derive from the preservation of structure.

In the present article we formulate, analyze, and implement EnergyEntropy-Momentum methods for discrete thermo-visco-elastic elements following the same ideas alluded to above. In the present case, the conserving/dissipative structure of the equations is relatively simple and one does not need to appeal to the underlying GENERIC formalism in order to split the evolution operators.

The thermo-visco-elastic elements are generalized discrete standard models in which the elastic and viscoelastic response is fully coupled with the temperature and serve as testbed for the development of numerical methods for more complex, three dimensional, thermo-visco-elastic material models. Also, these simplified discrete elements have interesting applications in 


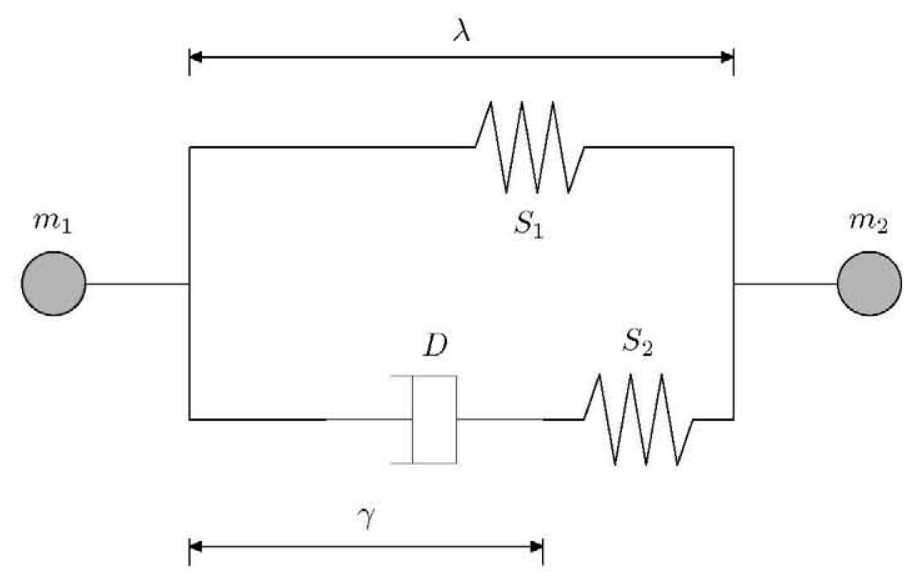

Figure 1: Thermo-visco-elastic element.

multibody dynamics. For example, the correct treatment of the thermal and viscous effects in dampers can be very important when modeling shock absorbers of vehicles traveling on rough surfaces (Lion and Loose, 2002). The proposed methods not only provide the correct and meaningful energy balance for the system but show, as expected, excellent robustness as compared with standard implicit methods. We must stress that the added stability is obtained without any artificial numerical damping which, although commonly used for multibody applications, often has the effect of breaking the symmetries of the problem and spoiling, for example, the conservation of angular momentum (Armero and Romero, 2001a).

The rest of the article is organized in the following way. In Section 2 the governing equations of the discrete thermo-visco-elastic element are presented. They are based on the finite strain thermo-visco-elastic model proposed in (Holzapfel and Simó, 1996) and are shown to be consistent with the two laws of thermodynamics. Then, a numerical method for integrating these equations is presented in Section 3. The algorithm preserves the fundamental laws identified in the continuum model as well as possible symmetries in the equations. The numerical examples of Section 4 serve to illustrate the conservation/dissipation features of the new method and compare it with standard implicit methods. The article closes with a summary of results in Section 5 . 


\section{The discrete thermo-visco-elastic model}

In this section we formulate the dynamic equations of a single discrete thermo-visco-elastic element, verifying that such evolution equations comply with the two laws of thermodynamics.

For that, consider an element such as the one depicted in Figure 1 consisting of one thermoelastic spring $S_{1}$ in parallel with another thermoelastic spring $S_{2}$, this one in series with a thermo-viscous dashpot $D$. This is the simplest generalization of the standard solid element that encompasses thermal coupling. Simpler models might be obtained by restricting, for example, the thermal coupling to the viscous element. Moreover, by setting the viscosity to zero one recovers the simpler discrete thermoelastic element.

At each of the two ends of the element, a point mass of value $m_{\alpha}$ is placed $(\alpha=1,2)$. If the position at time $t$ of each of these masses is denoted $\mathbf{q}_{\alpha}(t)$, then the corresponding momenta is $\mathbf{p}_{\alpha}=m_{\alpha} \dot{\mathbf{q}}_{\alpha}$, where the superposed dot indicates the time derivative. We consider also the possibility of external forces $\mathbf{f}_{\alpha}$ acting on each of the point masses. The temperature of the whole element is assumed to be unique and denoted by $\theta$, the length of the element is $\lambda=\left|\mathbf{q}_{1}-\mathbf{q}_{2}\right|$ and the stretch of the dashpot is denoted by $\gamma$. Internal heat flow is not considered, and the environment is considered to be a thermal reservoir at constant temperature.

To completely define the thermodynamic state of the element we propose the following free energy function

$$
\psi(\lambda, \theta, \gamma)=\psi^{\infty}(\lambda, \theta)+\Gamma(\lambda, \theta, \gamma)
$$

The functions $\psi^{\infty}$ and $\Gamma$ represent, respectively, the free energy of the spring $S_{1}$ in Figure 1 and the Maxwell element consisting of $S_{2}$ and $D$. Following the ideas presented in (Holzapfel and Simó, 1996) we restrict the functional form of the free energy $\Gamma$ to be

$$
\Gamma(\lambda, \theta, \gamma)=\beta \psi^{\infty}(\lambda, \theta)+\mu(\theta) \gamma^{2}-\beta \gamma \frac{\partial \psi^{\infty}(\lambda, \theta)}{\partial \lambda}
$$

with $\beta>0, \mu>0$. The basic motivation of selecting and presenting the specific format of the free energy function given by expressions (1) and (2) is to early introduce a model that has a direct rheological interpretation that may prove helpful to devise applications to real spring-damper assemblies.

From the general expression (1), the total internal force $f$, the force $g$ due to the dashpot, and the entropy $s$ of the element can be calculated by 
the expressions:

$$
f=\frac{\partial \psi}{\partial \lambda}, \quad g=-\frac{\partial \psi}{\partial \gamma}, \quad s=-\frac{\partial \psi}{\partial \theta} .
$$

The equation of balance of linear momentum can be stated, for each mass, as

$$
\dot{\mathbf{p}}_{\alpha}=-\frac{\partial \psi}{\partial \mathbf{q}_{\alpha}}+\mathbf{f}_{\alpha}=-\frac{\partial \psi}{\partial \lambda} \frac{\partial \lambda}{\partial \mathbf{q}_{\alpha}}+\mathbf{f}_{\alpha}=-f \frac{\partial \lambda}{\partial \mathbf{q}_{\alpha}}+\mathbf{f}_{\alpha} .
$$

The last balance law to be considered is the balance of energy of the element. Let $h$ be the heat flowing out of the element into the environment and $e=\psi+\theta s$ be its internal energy. Then, the balance of energy states that the power $f \dot{\lambda}$ exerted on the element is employed to change its internal energy or transferred into the environment through heat:

$$
f \dot{\lambda}=\dot{e}+h=\frac{\partial \psi}{\partial \lambda} \dot{\lambda}+\frac{\partial \psi}{\partial \theta} \dot{\theta}+\frac{\partial \psi}{\partial \gamma} \dot{\gamma}+\dot{\theta} s+\theta \dot{s}+h
$$

Using the definitions (3), the balance of energy can be simplified to

$$
\theta \dot{s}=g \dot{\gamma}-h
$$

To close the equations that define the response of the element, an evolution equation of the viscous deformation must be given. The simplest one is

$$
\dot{\gamma}=\frac{1}{\eta} g
$$

where $\eta=2 \mu \tau$ is the viscosity of the dashpot, and $\tau$ is a relaxation time. To summarize, the complete set of evolution equations of the thermo-viscoelastic element is

$$
\begin{aligned}
\dot{\mathrm{q}}_{\alpha} & =\frac{1}{m_{\alpha}} \mathbf{p}_{\alpha}, \\
\dot{\mathbf{p}}_{\alpha} & =-f \frac{\partial \lambda}{\partial \mathbf{q}_{\alpha}}+\mathbf{f}_{\alpha}, \\
\dot{\gamma} & =\frac{1}{\eta} g \\
\dot{s} & =\frac{1}{\theta}\left(\frac{g^{2}}{\eta}-h\right) .
\end{aligned}
$$

We check that the discrete model defined by equations (1) to (8) satisfies the entropy production inequality in the Clausius-Plank form. This amounts 
to the requirement that the dissipation $\mathcal{D}=f \dot{\lambda}-\dot{\psi}-s \dot{\theta}$ must be non-negative. Using the chain rule and the equations of the model we find that

$$
\begin{aligned}
\mathcal{D} & =f \dot{\lambda}-\dot{\psi}-s \dot{\theta} \\
& =f \dot{\lambda}-\frac{\partial \psi}{\partial \lambda} \dot{\lambda}-\frac{\partial \psi}{\partial \theta} \dot{\theta}-\frac{\partial \psi}{\partial \gamma} \dot{\gamma}-s \dot{\theta} \\
& =g \dot{\gamma} \\
& =\frac{1}{\eta} g^{2},
\end{aligned}
$$

which is non-negative and, moreover, vanishes only when the viscous force is zero.

\subsection{The first law of thermodynamics}

The first law of thermodynamics postulates that the power supplied to a system (either by mechanical or thermal means) is employed in changing its total energy (internal plus kinetic). We verify next that the model previously defined is consistent with this fundamental principle.

For a thermo-visco-elastic element possibly connected to other mechanical systems, the power exerted by forces acting on it and the thermal power received are defined, respectively as:

$$
\begin{aligned}
P_{m e c} & =\sum_{\alpha=1}^{2} \mathbf{f}_{\alpha} \cdot \dot{\mathbf{q}}_{\alpha}, \\
P_{t h} & =-h .
\end{aligned}
$$

The total energy of the same element is $E=K+e$, the sum of the internal energy $e$, previously defined, and the kinetic energy

$$
K=\frac{1}{2} \sum_{\alpha=1}^{2} \frac{\mathrm{p}_{\alpha} \cdot \mathrm{p}_{\alpha}}{m_{\alpha}}
$$

With this notation, the first law of thermodynamics reads

$$
P_{m e c}+P_{t h}=\dot{E} \text {. }
$$

Using the definitions of the kinetic and internal energies, the time derivative of the total energy can be calculated:

$$
\dot{E}=\sum_{\alpha=1}^{2} \frac{1}{m_{\alpha}} \mathbf{p}_{\alpha} \cdot \dot{\mathbf{p}}_{\alpha}+\frac{\partial \psi}{\partial \lambda} \dot{\lambda}+\frac{\partial \psi}{\partial \theta} \dot{\theta}+\frac{\partial \psi}{\partial \gamma} \dot{\gamma}+\dot{s} \theta+s \dot{\theta}
$$


Employing the definitions of momentum, internal force, entropy, force in the dashpot, and the chain rule, the previous equation simplifies to

$$
\dot{E}=\sum_{\alpha=1}^{2} \dot{\mathbf{q}}_{\alpha} \cdot \dot{\mathbf{p}}_{\alpha}+\sum_{\alpha=1}^{2} f \frac{\partial \lambda}{\partial \mathbf{q}_{\alpha}} \cdot \dot{\mathbf{q}}_{\alpha}-g \dot{\gamma}+\dot{s} \theta=\sum_{\alpha=1}^{2}\left(\dot{\mathbf{p}}_{\alpha}+f \frac{\partial \lambda}{\partial \mathbf{q}_{\alpha}}\right) \cdot \dot{\mathbf{q}}_{\alpha}-h,
$$

which, in view of equation $(8)_{2}$, amounts precisely to the sum of the external power supplied to element, proving the satisfaction of the first law.

\subsection{The second law of thermodynamics}

We next verify that system consisting of the element and the environment possess an non-decreasing entropy. In this work we only consider thermal exchange between thermo-visco-elastic elements and the environment, and thus it is unnecessary to study the entropy generated by heat conduction among possible interconnected mechanical elements.

In what follows, the environment is considered to be a thermal reservoir at constant temperature $\theta_{r}$. The total entropy $S$ at time $t$ of the system consisting of a thermo-visco-elastic element and the reservoir is defined to be

$$
S=s+\int_{0}^{t} \frac{h}{\theta_{r}} \mathrm{~d} t
$$

In addition to the evolution equations (8), the heat conduction between the mechanical element and the environment must be governed by an additional equation which we postulate to be

$$
h=c\left(\theta-\theta_{r}\right) \text {, }
$$

with $c>0$ being the thermal conductivity between the element and the environment. Different constants allow to consider different thermal insulations for the elements in the system.

The rate of change of the total entropy can be calculated using (8) 4 and (16)

$$
\dot{S}=\dot{s}+\frac{h}{\theta_{r}}=\frac{g^{2}}{\eta \theta}+h\left(\frac{1}{\theta_{r}}-\frac{1}{\theta}\right)=\frac{g^{2}}{\eta \theta}+\frac{c}{\theta \theta_{r}}\left(\theta_{r}-\theta\right)^{2},
$$

which is always non-negative, hence proving the second law of thermodynamics. We emphasize that this proof can be trivially extended to the case with several thermo-visco-elastic elements, since each of them exchanges heat with the environment independently. 


\subsection{Symmetries}

Noether's theorem indicates that every mechanical system has a conservation law for each symmetry on its evolution equations. In the system studied in this section, translational and rotational symmetries might appear and the corresponding conserved quantities would be the linear and angular momentum, defined respectively as

$$
\mathbf{L}=\sum_{\alpha=1}^{2} \mathbf{p}_{\alpha}, \quad \mathbf{J}=\sum_{\alpha=1}^{2} \mathbf{q}_{\alpha} \times \mathbf{p}_{\alpha}
$$

The classical results concerning the conservation of these momenta in the situations in which the resultant force and torque vanish, are valid for the proposed model. The proofs are omitted.

In this section we have formulation a model for a thermo-visco-elastic system that satisfies the laws of thermodynamics and exhibits additional conservation laws resulting from the symmetries of the equations. The evolution equations (8) can be solved numerically to integrate the state variables $\mathbf{q}_{\alpha}, \mathbf{p}_{\alpha}, \theta, \gamma$ in time. Instead of employing standard methods for this task we propose in the next section a novel time integration scheme that preserves all these qualitative features of the model.

\section{Consistent integration: energy-entropy method}

In this section we develop a numerical method to integration the system of ordinary differential equations 8 . The salient feature of the proposed method is that it preserves all the conservation laws identified in Section 2.

The point of departure for obtaining the set of discrete evolution equations is to express (8) in entropy form. In order to do that, we apply the Legendre transformation to the free energy function $\psi(\lambda, \gamma, \theta)$ with respect to the temperature $\theta$. The conjugated variable is the entropy $s$ and the transformed function is the internal energy function $e(\lambda, \gamma, s)$ :

$$
s=-\frac{\partial \psi(\lambda, \gamma, \theta)}{\partial \theta} \quad, \quad e(\lambda, \gamma, s)=\psi(\lambda, \gamma, \hat{\theta}(\lambda, \gamma, s))+\hat{\theta}(\lambda, \gamma, s) s
$$

where $\hat{\theta}(\lambda, \gamma, s)$ is the temperature function obtained by the inversion of $(19)_{1}$. Either by applying the properties of the Legendre transform or just by using relations (3), it is follows that:

$$
f=\frac{\partial e}{\partial \lambda}, \quad g=-\frac{\partial e}{\partial \gamma}, \quad \theta=\frac{\partial e}{\partial s} .
$$


A key ingredient of the formulation is the use of the discrete derivative in a partitioned case, as introduced in (González, 1996). For our purposes, let us consider a smooth function $f: \mathbb{R}^{N} \rightarrow \mathbb{R}$ with $N \geq 1$. A second-order accurate discrete derivative $\mathrm{D} f: \mathbb{R}^{N} \times \mathbb{R}^{N} \rightarrow \mathbb{R}^{N}$ is defined by:

$$
\mathrm{D} f(\boldsymbol{x}, \boldsymbol{y}) \cdot \boldsymbol{u}=\sum_{i=1}^{N} \mathrm{D}^{i} f\left(x_{i}, y_{i}\right) \cdot u_{i},
$$

where $\boldsymbol{x}, \boldsymbol{y} \in \mathbb{R}^{N}$ and $\boldsymbol{u} \in \mathbb{R}^{N}$. The terms $\mathrm{D}^{i} f\left(x_{i}, y_{i}\right)$ may be interpreted as approximations to the partial derivatives of $f$ respect to the $i$-th variable. $\mathrm{D} f(\boldsymbol{x}, \boldsymbol{y})$ satisfies the directionality and consistency conditions of the discrete derivative, namely:

$$
\begin{aligned}
\mathrm{D} f(\boldsymbol{x}, \boldsymbol{y}) \cdot(\boldsymbol{y}-\boldsymbol{x}) & =f(\boldsymbol{y})-f(\boldsymbol{x}) \\
\mathrm{D} f(\boldsymbol{x}, \boldsymbol{y}) & =D f\left(\frac{\boldsymbol{x}+\boldsymbol{y}}{2}\right)+\mathcal{O}\left(|\boldsymbol{y}-\boldsymbol{x}|^{2}\right),
\end{aligned}
$$

for all $\boldsymbol{x}, \boldsymbol{y} \in \mathbb{R}^{N}$. The operator $D$ in (23) denotes the standard derivative. One of the properties of the discrete derivative that is employed in what follows is a discrete version of the chain rule. As proved in (González, 1996, $\operatorname{pg} 75$ ), given functions $f: \mathbb{R}^{N} \rightarrow \mathbb{R}, \tilde{f}: \mathbb{R}^{M} \rightarrow \mathbb{R}$, and $\pi: \mathbb{R}^{N} \rightarrow \mathbb{R}^{M}$, with $N>M$ and $f=\tilde{f} \circ \pi$, then if $\pi$ is at most of degree two, the following chain rule applies:

$$
\mathrm{D} f(\boldsymbol{x}, \boldsymbol{y})=D \pi\left(\frac{\boldsymbol{x}+\boldsymbol{y}}{2}\right)^{T} \mathrm{D} \tilde{f}(\pi(\boldsymbol{x}), \pi(\boldsymbol{y})) .
$$

We consider next the formulation of a time-stepping scheme for the evolution equations (8) in a time interval $[0, T]$. For that, we consider a partition of the integration interval into subintervals of the form $\left(t_{n}, t_{n+1}\right)$ of length $\Delta t_{n}=t_{n+1}-t_{n}$ which, for simplicity, is assumed to be constant and denoted simply as $\Delta t$. Then the proposed time integration method is given by the 
algebraic system of equations:

$$
\begin{aligned}
\frac{\mathbf{q}_{1, n+1}-\mathbf{q}_{1, n}}{\Delta t} & =\frac{1}{m_{1}} \mathbf{p}_{1, n+1 / 2} \\
\frac{\mathbf{q}_{2, n+1}-\mathbf{q}_{2, n}}{\Delta t} & =\frac{1}{m_{2}} \mathbf{p}_{2, n+1 / 2} \\
\frac{\mathbf{p}_{1, n+1}-\mathbf{p}_{1, n}}{\Delta t} & =-f^{*} \frac{\left(\mathbf{q}_{1}-\mathbf{q}_{2}\right)_{n+1 / 2}}{\hat{\lambda}_{n+1 / 2}}+\tilde{\mathbf{f}}_{1} \\
\frac{\mathbf{p}_{2, n+1}-\mathbf{p}_{2, n}}{\Delta t} & =-f^{*} \frac{\left(\mathbf{q}_{2}-\mathbf{q}_{1}\right)_{n+1 / 2}}{\hat{\lambda}_{n+1 / 2}}+\tilde{\mathbf{f}}_{2} \\
\frac{\gamma_{n+1}-\gamma_{n}}{\Delta t} & =\frac{1}{\eta^{*}} g^{*} \\
\frac{s_{n+1}-s_{n}}{\Delta t} & =\frac{1}{\theta^{*}}\left(\frac{g^{*}}{\eta^{*}}-h^{*}\right)
\end{aligned}
$$

where $\hat{\lambda}_{n+1 / 2}=\left|\mathbf{q}_{1, n+1 / 2}-\mathbf{q}_{2, n+1 / 2}\right|$ and $(\cdot)_{n+1 / 2}=\frac{1}{2}(\cdot)_{n}+\frac{1}{2}(\cdot)_{n+1}$.

The terms $\tilde{\mathbf{f}}_{1}$ and $\tilde{\mathbf{f}}_{2}$ are second-order approximations of the external forces at the corresponding nodes, and the terms $f^{*}, g^{*}$ and $\theta^{*}$ are second-order discrete approximations of the total internal force, the dashpot force and the temperature, respectively, given by:

$$
\begin{aligned}
& f^{*}=\mathrm{D}^{\lambda} e\left(\lambda_{n}, \lambda_{n+1}\right), \\
& g^{*}=-\mathrm{D}^{\gamma} e\left(\gamma_{n}, \gamma_{n+1}\right), \\
& \theta^{*}=\mathrm{D}^{s} e\left(s_{n}, s_{n+1}\right) .
\end{aligned}
$$

The terms related to viscosity and heat conduction are:

$$
\eta^{*}=\eta\left(\theta^{*}\right), \quad h^{*}=c\left(\theta^{*}-\theta_{r}\right) .
$$

Note the parallelism between the continuous expressions (20) and their discrete counterparts (26).

Elaborated expressions for (26) can be obtained according to (González, 1996, pg 75), resulting in: 


$$
\begin{aligned}
\mathrm{D}^{\lambda} e\left(\lambda_{n}, \lambda_{n+1}\right) & =\frac{e\left(\lambda_{n+1}, \gamma_{n+1}, s_{n+1}\right)-e\left(\lambda_{n}, \gamma_{n+1}, s_{n+1}\right)}{2\left(\lambda_{n+1}-\lambda_{n}\right)} \\
& +\frac{e\left(\lambda_{n+1}, \gamma_{n}, s_{n}\right)-e\left(\lambda_{n}, \gamma_{n}, s_{n}\right)}{2\left(\lambda_{n+1}-\lambda_{n}\right)} \\
\mathrm{D}^{\gamma} e\left(\gamma_{n}, \gamma_{n+1}\right) & =\frac{e\left(\lambda_{n}, \gamma_{n+1}, s_{n+1}\right)-e\left(\lambda_{n}, \gamma_{n}, s_{n+1}\right)}{2\left(\gamma_{n+1}-\gamma_{n}\right)} \\
& +\frac{e\left(\lambda_{n+1}, \gamma_{n+1}, s_{n}\right)-e\left(\lambda_{n+1}, \gamma_{n}, s_{n}\right)}{2\left(\gamma_{n+1}-\gamma_{n}\right)} \\
\mathrm{D}^{s} e\left(\gamma_{n}, s_{n+1}\right) & =\frac{e\left(\lambda_{n}, \gamma_{n}, s_{n+1}\right)-e\left(\lambda_{n}, \gamma_{n}, s_{n}\right)}{2\left(s_{n+1}-s_{n}\right)} \\
& +\frac{e\left(\lambda_{n+1}, \gamma_{n+1}, s_{n+1}\right)-e\left(\lambda_{n+1}, \gamma_{n+1}, s_{n}\right)}{2\left(s_{n+1}-s_{n}\right)}
\end{aligned}
$$

In the limit case $\lambda_{n+1}=\lambda_{n}, \gamma_{n+1}=\gamma_{n}$ and $s_{n+1}=s_{n}$, the previous discrete derivatives simplify to:

$$
\begin{aligned}
\mathrm{D}^{\lambda} e\left(\lambda_{n}, \lambda_{n}\right) & =\frac{1}{2}\left[D^{\lambda} e\left(\lambda_{n}, \gamma_{n+1}, s_{n+1}\right)+D^{\lambda} e\left(\lambda_{n}, \gamma_{n}, s_{n}\right)\right] \\
\mathrm{D}^{\gamma} e\left(\gamma_{n}, \gamma_{n}\right) & =\frac{1}{2}\left[D^{\gamma} e\left(\lambda_{n}, \gamma_{n}, s_{n+1}\right)+D^{\gamma} e\left(\lambda_{n+1}, \gamma_{n}, s_{n}\right)\right] \\
\mathrm{D}^{s} e\left(s_{n}, s_{n}\right) & =\frac{1}{2}\left[D^{s} e\left(\lambda_{n}, \gamma_{n}, s_{n}\right)+D^{s} e\left(\lambda_{n+1}, \gamma_{n+1}, s_{n}\right)\right]
\end{aligned}
$$

In the next sections it will be shown that the time stepping (25) exactly satisfies the first and second laws of thermodynamics in a discrete form.

\subsection{The first law of thermodynamics in discrete form}

The discrete version of the first law states that the balance of external work (mechanical or thermal) equals the balance of total energy (internal plus kinetic) in a time step for the element. We verify next that the model previously defined is consistent with this fundamental principle.

In order to check this assertion, let us denote by $\boldsymbol{z}=\left(\mathbf{q}_{1}, \mathbf{q}_{2}, \mathbf{p}_{1}, \mathbf{p}_{2}, \gamma, s\right)$ the element state and introduce the notation:

$$
\mathrm{D}^{\mathbf{v}}\left(\mathbf{v}_{n}, \mathbf{v}_{n+1}\right)=\left(\mathrm{D}^{v_{1}}\left(v_{1, n}, v_{1, n+1}\right), \ldots, \mathrm{D}^{v_{N}}\left(v_{N, n}, v_{N, n+1}\right)\right)
$$

for $\mathbf{v}=\left(v_{1}, \ldots, v_{N}\right) \in \mathbb{R}^{N}$. 
The balance of total energy of the element, based on the directionality property (22) of the discrete derivative in the partitioned case (21) reads:

$$
\begin{aligned}
E_{n+1}-E_{n} & =\mathrm{D} E\left(\boldsymbol{z}_{n}, \boldsymbol{z}_{n+1}\right) \cdot\left(\boldsymbol{z}_{n+1}-\boldsymbol{z}_{n}\right) \\
& =\mathrm{D}^{\mathbf{q}_{1}} E\left(\mathbf{q}_{1, n}, \mathbf{q}_{1, n+1}\right) \cdot\left(\mathbf{q}_{1, n+1}-\mathbf{q}_{1, n}\right) \\
& +\mathrm{D}^{\mathbf{q}_{2}} E\left(\mathbf{q}_{2, n}, \mathbf{q}_{2, n+1}\right) \cdot\left(\mathbf{q}_{2, n+1}-\mathbf{q}_{2, n}\right) \\
& +\mathrm{D}^{\mathbf{p}_{1}} E\left(\mathbf{p}_{1, n}, \mathbf{p}_{1, n+1}\right) \cdot\left(\mathbf{p}_{1, n+1}-\mathbf{p}_{1, n}\right) \\
& +\mathrm{D}^{\mathbf{p}_{2}} E\left(\mathbf{p}_{2, n}, \mathbf{p}_{2, n+1}\right) \cdot\left(\mathbf{p}_{2, n+1}-\mathbf{p}_{2, n}\right) \\
& +\mathrm{D}^{\gamma} E\left(\gamma_{n}, \gamma_{n+1}\right) \cdot\left(\gamma_{n+1}-\gamma_{n}\right) \\
& +\mathrm{D}^{s} E\left(s_{n}, s_{n+1}\right) \cdot\left(s_{n+1}-s_{n}\right)
\end{aligned}
$$

Taking into consideration the expression for the total energy $E=K+e$, rewritten here in order to highlight the dependence on the different terms on the state variables, we have:

$$
E=\frac{1}{2 m_{1}} \mathbf{p}_{1}^{2}+\frac{1}{2 m_{2}} \mathbf{p}_{2}^{2}+e\left(\lambda\left(\mathbf{q}_{1}, \mathbf{q}_{2}\right), \gamma, s\right) .
$$

The different terms of the previous expression may be further elaborated using the definition of the discrete derivative and the chain rule (24):

$$
\begin{aligned}
\mathrm{D}^{\mathbf{q}_{1}} E\left(\mathbf{q}_{1, n}, \mathbf{q}_{1, n+1}\right) & =\mathrm{D}^{\lambda} e\left(\lambda_{n}, \lambda_{n+1}\right) \frac{\left(\mathbf{q}_{1}-\mathbf{q}_{2}\right)_{n+1 / 2}}{\hat{\lambda}_{n+1 / 2}} \\
& =f^{*} \frac{\left(\mathbf{q}_{1}-\mathbf{q}_{2}\right)_{n+1 / 2}}{\hat{\lambda}_{n+1 / 2}} \\
\mathrm{D}^{\mathbf{q}_{2}} E\left(\mathbf{q}_{2, n}, \mathbf{q}_{2, n+1}\right) & =\mathrm{D}^{\lambda} e\left(\lambda_{n}, \lambda_{n+1}\right) \frac{\left(\mathbf{q}_{2}-\mathbf{q}_{1}\right)_{n+1 / 2}}{\hat{\lambda}_{n+1 / 2}} \\
& =f^{*} \frac{\left(\mathbf{q}_{2}-\mathbf{q}_{1}\right)_{n+1 / 2}}{\hat{\lambda}_{n+1 / 2}} \\
\mathrm{D}^{\mathbf{p}_{1}} E\left(\mathbf{p}_{1, n}, \mathbf{p}_{1, n+1}\right) & =\frac{\mathbf{p}_{1_{n+1 / 2}}}{m_{1}} \\
\mathrm{D}^{\mathbf{p}_{2}} E\left(\mathbf{p}_{2, n}, \mathbf{p}_{2, n+1}\right) & =\frac{\mathbf{p}_{2_{n+1 / 2}}}{m_{1}} \\
\mathrm{D}^{\gamma} E\left(\gamma_{n}, \gamma_{n+1}\right) & =\mathrm{D}^{\gamma} e\left(\gamma_{n}, \gamma_{n+1}=-g^{*}\right. \\
\mathrm{D}^{s} E\left(s_{n}, s_{n+1}\right) & =\mathrm{D}^{s} e\left(s_{n}, s_{n+1}=\theta^{*}\right.
\end{aligned}
$$


Further manipulation of relations (31) using (33) and the discrete evolution equations (25) leads to:

$$
\begin{aligned}
E_{n+1}-E_{n} & =\left(\tilde{\mathbf{f}}_{1}-\frac{\mathbf{p}_{1, n+1}-\mathbf{p}_{1, n}}{\Delta t}\right) \cdot\left(\mathbf{q}_{1, n+1}-\mathbf{q}_{1, n}\right) \\
& +\left(\tilde{\mathbf{f}}_{2}-\frac{\mathbf{p}_{2, n+1}-\mathbf{p}_{2, n}}{\Delta t}\right) \cdot\left(\mathbf{q}_{2, n+1}-\mathbf{q}_{2, n}\right) \\
& +\frac{\mathbf{q}_{1, n+1}-\mathbf{q}_{1, n}}{\Delta t} \cdot\left(\mathbf{p}_{1, n+1}-\mathbf{p}_{1, n}\right) \\
& +\frac{\mathbf{q}_{2, n+1}-\mathbf{q}_{2, n}}{\Delta t} \cdot\left(\mathbf{p}_{2, n+1}-\mathbf{p}_{2, n}\right) \\
& -g^{*} \frac{\Delta t}{\eta^{*}} g^{*}+\theta^{*} \frac{\Delta t}{\theta^{*}}\left(\frac{g^{*^{2}}}{\eta^{*}}-h^{*}\right) \\
& =\tilde{\mathbf{f}}_{1} \cdot\left(\mathbf{q}_{1, n+1}-\mathbf{q}_{1, n}\right)+\tilde{\mathbf{f}}_{2} \cdot\left(\mathbf{q}_{2, n+1}-\mathbf{q}_{2, n}\right)-h^{\star} \Delta t
\end{aligned}
$$

which proves the second-order approximation of the energy balance of the element.

An alternative result can be obtained applying the first law to the entire system (element plus the environment). In this case, the first principle states that the time derivative of the total energy is just the external mechanical power supplied to the system. The discrete version of this result is obtained considering the following discrete evolution equation for the ambient entropy $\sigma:$

$$
\frac{\sigma_{n+1}-\sigma_{n}}{\Delta t}=\frac{h^{*}}{\theta_{r}}
$$

with $h^{*}$ given by expression $(27)_{2}$. Using this equation, the ambient energy balance results:

$$
E_{n+1}^{a m b}-E_{n}^{a m b}=\theta_{r}\left(\sigma_{n+1}-\sigma_{n}\right)=h^{*} \Delta t,
$$

and employing (34) is immediately obtained that the energy balance equals the external mechanical work. A corollary of this result is that the total discrete energy is exactly constant provided that the external forces vanish.

\subsection{The second law of thermodynamics in discrete form}

We must verify that the discrete total entropy of the system never decreases. Using the discrete evolution equations (25) and (35), the balance of 
total entropy results in:

$$
\begin{aligned}
S_{n+1}-S_{n} & =\left(s_{n+1}-s_{n}\right)+\left(\sigma_{n+1}-\sigma_{n}\right) \\
& =\frac{\Delta t}{\theta^{*}}\left(\frac{g^{*^{2}}}{\eta^{*}}-h^{*}\right)+\frac{\Delta t h^{*}}{\theta_{r}},
\end{aligned}
$$

which is non-negative provided that relation $(27)_{2}$ is verified.

\subsection{Discrete symmetries}

Finally, it is possible to prove that the discrete scheme possesses the translational and rotational symmetries of the continuous evolution problem. Let us denote by $\mathrm{f}^{*}$ the consistent internal force of the element, given by:

$$
\mathbf{f}^{*}=f^{*} \frac{\left(\mathrm{q}_{1}-\mathrm{q}_{2}\right)_{n+1 / 2}}{\hat{\lambda}_{n+1 / 2}}
$$

Taking into account the relations $(25)_{3,4}$, the balance of linear momentum results:

$\mathbf{L}_{n+1}-\mathbf{L}_{n}=\sum_{\alpha=1}^{2}\left(\mathbf{p}_{\alpha, n+1}-\mathbf{p}_{\alpha, n}\right)=\Delta t\left[\left(-\mathbf{f}^{*}+\tilde{\mathbf{f}}_{1}\right)+\left(\mathbf{f}^{*}+\tilde{\mathbf{f}}_{2}\right)\right]=\Delta t \sum_{\alpha=0}^{2} \tilde{\mathbf{f}}_{\alpha}$,

which proves the exact conservation of the linear momentum for a force-free motion.

On the other hand, a convenient point of departure for the evaluation of the angular momentum balance is the following expression:

$\mathbf{J}_{n+1}-\mathbf{J}_{n}=\sum_{\alpha=0}^{2}\left[\mathbf{q}_{\alpha, n+1 / 2} \times\left(\mathbf{p}_{\alpha, n+1}-\mathbf{p}_{\alpha, n}\right)\right]-\left[\mathbf{p}_{\alpha, n+1 / 2} \times\left(\mathbf{q}_{\alpha, n+1}-\mathbf{q}_{\alpha, n}\right)\right]$

Using expressions $(25)_{1,2}$ is immediately verified that $\mathbf{p}_{\alpha, n+1 / 2} \times\left(\mathbf{q}_{\alpha, n+1}-\right.$ $\left.\mathbf{q}_{\alpha, n}\right)=0$ for $\alpha=1,2$. Employing relations (40) and $(25)_{3,4}$ with the definition (38) it follows that:

$$
\begin{aligned}
\mathbf{J}_{n+1}-\mathbf{J}_{n} & =\sum_{\alpha=0}^{2} \mathbf{q}_{\alpha, n+1 / 2} \times\left(\mathbf{p}_{\alpha, n+1}-\mathbf{p}_{\alpha, n}\right) \\
& =\Delta t\left(\mathbf{q}_{2}-\mathrm{q}_{1}\right)_{n+1 / 2} \times \mathbf{f}^{*}+\Delta t \sum_{\alpha=0}^{2} \mathbf{q}_{\alpha, n+1 / 2} \times \tilde{\mathbf{f}}_{\alpha} .
\end{aligned}
$$


Finally, using the definition (38) it is verified that the first term of (42) vanishes and the exact conservation of angular momentum for a force-free motion is obtained.

\section{Numerical simulations}

We consider in this section two representative simulations which will illustrate the performance of the proposed formulation, comparing it with other standard integration schemes.

\subsection{Single particle system}

Let us consider a mechanical system consisting of a particle of mass $m$ connected by a thermo-visco-elastic element to a fixed point of space. The free energy of the main spring is given by:

$$
\psi^{\infty}(\lambda, \theta)=\frac{c_{1}(\theta)}{2} \log ^{2} \frac{\lambda}{\lambda_{0}}-\beta_{c}\left(\theta-\theta_{r}\right) \log \frac{\lambda}{\lambda_{0}}+c_{0}\left(\theta-\theta_{r}-\theta \log \frac{\theta}{\theta_{r}}\right),
$$

where $\theta_{b}$ is the ambient temperature, $\beta_{c}$ is the thermo-elastic coupling parameter and $c_{0}$ is the heat capacity. The format of the free energy function is taken from (Romero, 2009) and accounts for nonlinear (logarithmic) deformations, a thermomechanical Gough-Joule type coupling and dilatation in the first, second and third terms respectively.

The term $c_{1}$ is assumed to have a linear dependence with the temperature, given by $c_{1}(\theta)=c_{10}-c_{11}\left(\theta-\theta_{r}\right)$, with $c_{10}, c_{11}>0$. The parameter $\mu$ associated with the free energy function of the Maxwell element (2) is assumed to have also a linear dependence with the temperature, given by $\mu(\theta)=\mu_{0}-\mu_{1}\left(\theta-\theta_{r}\right)$, with $\mu_{0}, \mu_{1}>0$. Finally, parameter $\beta$ in (2) is assumed to relate the stiffness of the associated linear springs at the ambient temperature, $\beta=2 \mu_{0} / c_{10}$.

The damping parameter $\eta$ of the damper is assumed to follow an exponential law:

$$
\eta(\theta)=\eta_{0} \exp \left[a\left(\frac{1}{\theta}-\frac{1}{\theta_{r}}\right)\right] \quad \text { with } \quad \eta_{0}, a>0
$$

The dependency of the damping with the temperature is very important for realistic simulations of some practical systems; for instance, for the accurate dynamical characterization of vehicles equipped with oil shock absorbers on 


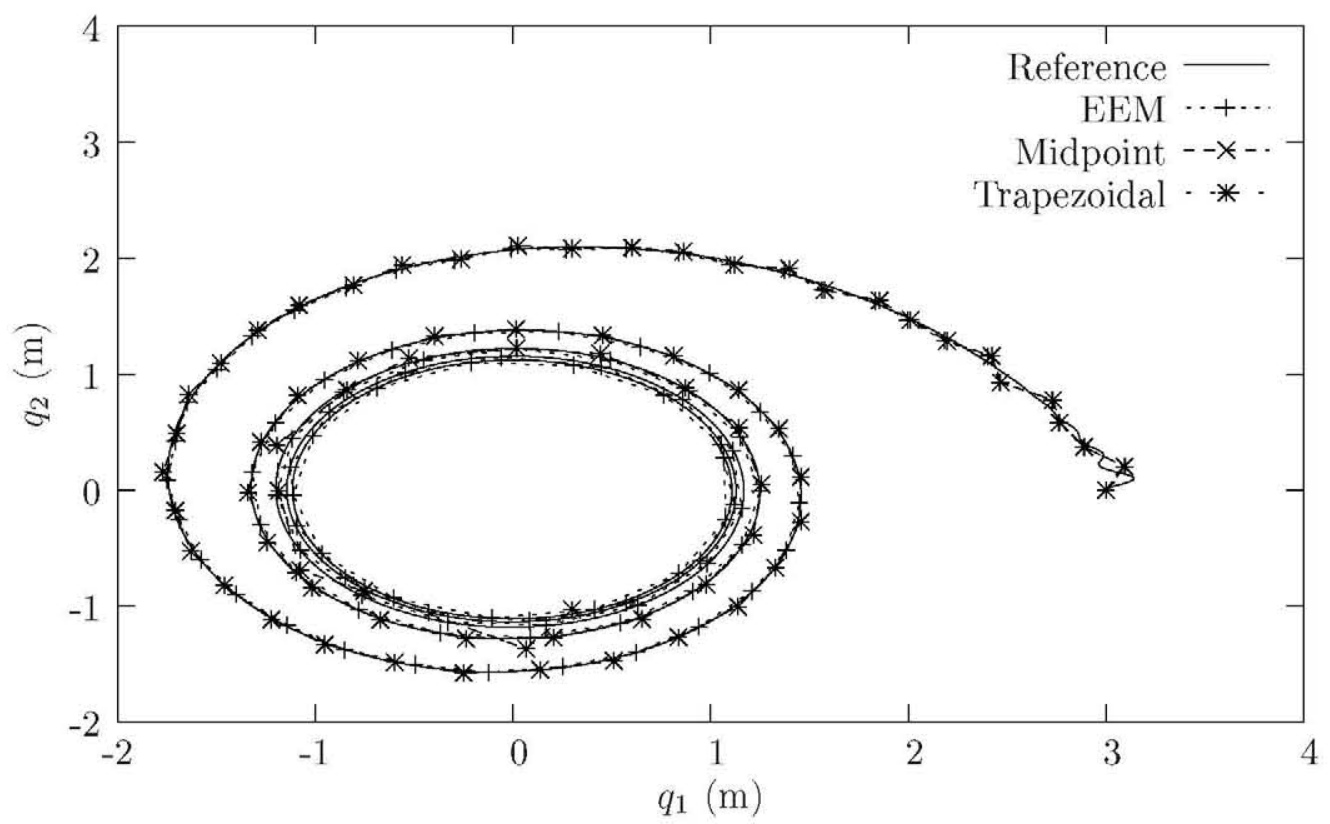

Figure 2: Trajectory

long and rugged tracks. In this case, an increment of the shock absorber temperature produces a reduction of the oil viscosity, influencing the handling and comfort behavior. Experimental force/velocity curves in the literature of automotive shock absorbers (see, for example, Lion and Loose (2002)) follow quite accurately exponential laws such as (44).

The data employed in the simulation is $m=1 \mathrm{~kg}, \lambda_{0}=1 \mathrm{~m}, \theta_{r}=300$ $\mathrm{K}, c_{10}=100 \mathrm{Nm}, c_{11}=0.5 \mathrm{NmK}^{-1}, \beta_{c}=4 \mathrm{NmK}^{-1}, c_{0}=1 \mathrm{NmK}^{-1}, \mu_{0}=5$ $\mathrm{Nm}^{-1}, \mu_{1}=0.1 \mathrm{Nm}^{-1} \mathrm{~K}^{-1}, \eta_{0}=100 \mathrm{Nsm}^{-1}, a=10 \mathrm{~K}, c=10 \mathrm{Nms}^{-1} \mathrm{~K}^{-1}$. The motion is integrated up to $20 \mathrm{~s}$ with a constant time step size $\Delta t=0.2$ $\mathrm{s}$ and initial conditions:

$$
\mathbf{q}_{0}=[3,0] \mathrm{m} \quad, \quad \dot{\mathbf{q}}_{0}=[0,1] \mathrm{m} / \mathrm{s} \quad, \quad \gamma_{0}=0 \mathrm{~m} \quad, \quad \theta_{0}=380 \mathrm{~K} .
$$

The solution of the evolution equations in entropy form is obtained with three methods: the standard midpoint rule, the trapezoidal rule and the energy-entropy method (EEM). What is more, a reference solution is computed with the midpoint rule and a timestep $\Delta t_{r e f}=0.005 \mathrm{~s}$. Figure 2 shows the trajectory of the particle as obtained with all methods. The integration with the midpoint rule and the trapezoidal rule blows up at $t=14 \mathrm{~s}$ and 


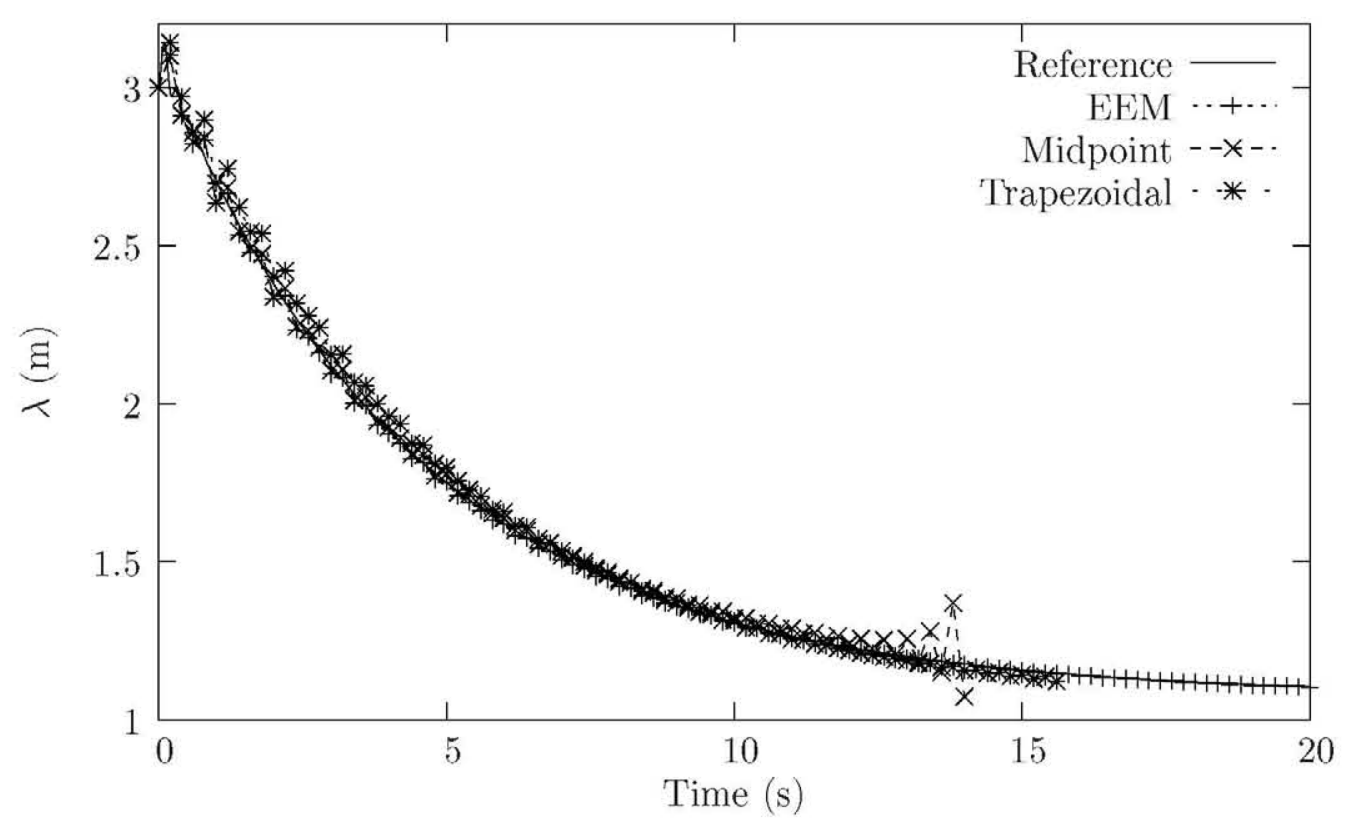

Figure 3: Length of the element $\lambda$ vs. time

$t=15.7 \mathrm{~s}$, respectively, but the consistent method correctly integrates the motion as it approaches a relative equilibrium.

Figure 3 show the evolution of the length of the element $(\lambda)$, Figure 4 the internal variable $(\gamma)$ and Figure 5 the evolution of the temperature $(\theta)$. All the curves associated with the midpoint integration show large oscillations developing long ahead the definitive blow up of the computation. On the other hand, the trapezoidal rule shows a smoother behavior but a more abrupt failure, with no warning. Figure 6 shows the evolution of the angular momentum, which is exactly constant for both the midpoint rule and the consistent integration, and totally erratic for the trapezoidal rule. Figures 7 indicate that the failure of the midpoint integration is related with a positive growth of the total energy, violating the first law of thermodynamics. The same comment can be made about the trapezoidal rule. On the other hand, the consistent integrator produces a constant total energy, which exactly agrees with the behavior of the original evolution equations (8).

Finally, Figure 8 shows the evolution of the total entropy. Both the midpoint and the trapezoidal rules produce a solution in which the total entropy apparently never decreases, although there are small oscillations in 


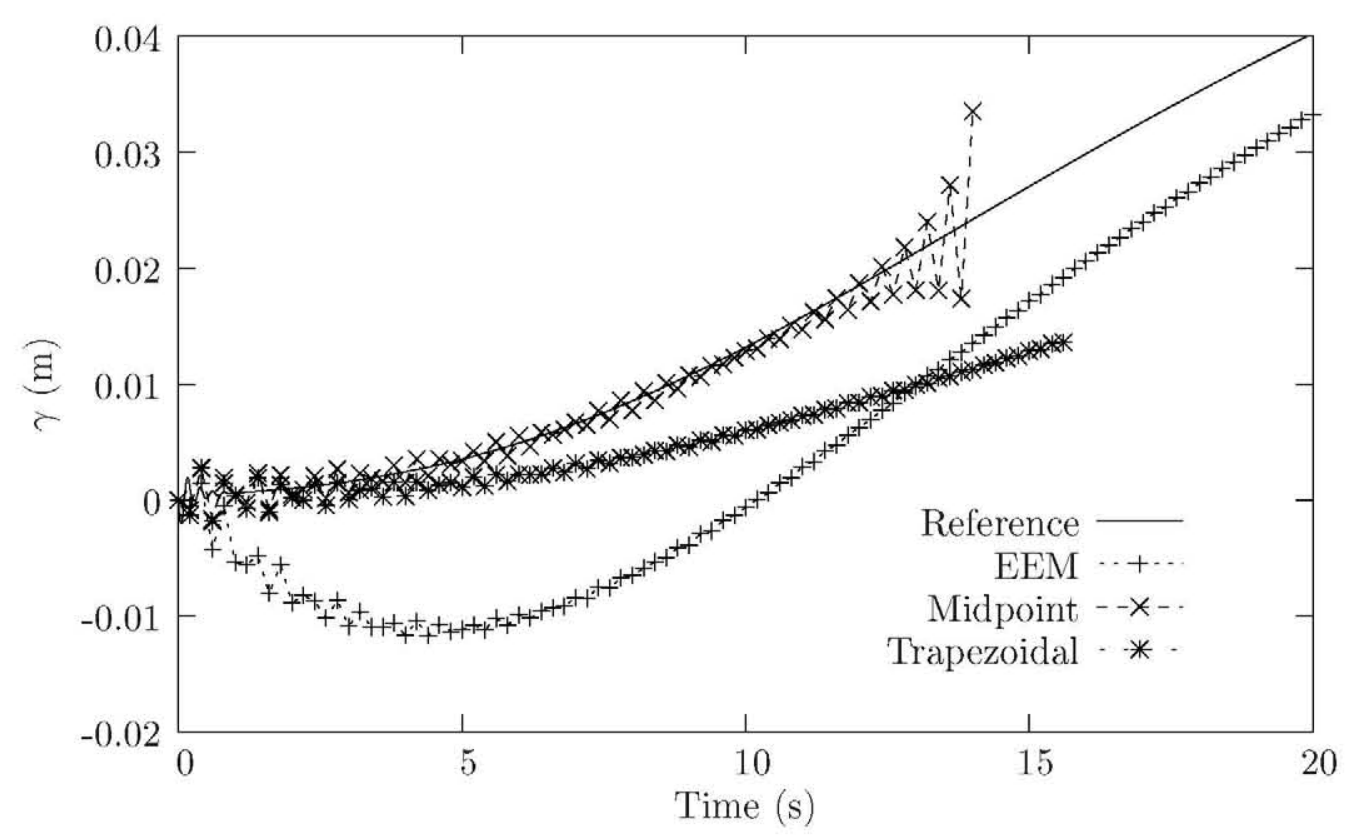

Figure 4: Viscous stretch $\gamma$ vs. time

its evolution. This qualitative feature can not be guaranteed and, in fact, does not hold when larger time step sizes are selected.

Note that the failure of the midpoint rule documented in this example could be avoided reducing the integration time step. The apparent superiority of the EEM is mainly related to stability, while exactly complying with the laws of thermodynamics and the symmetries of the original system, which improves the reliability of the obtained solution. 


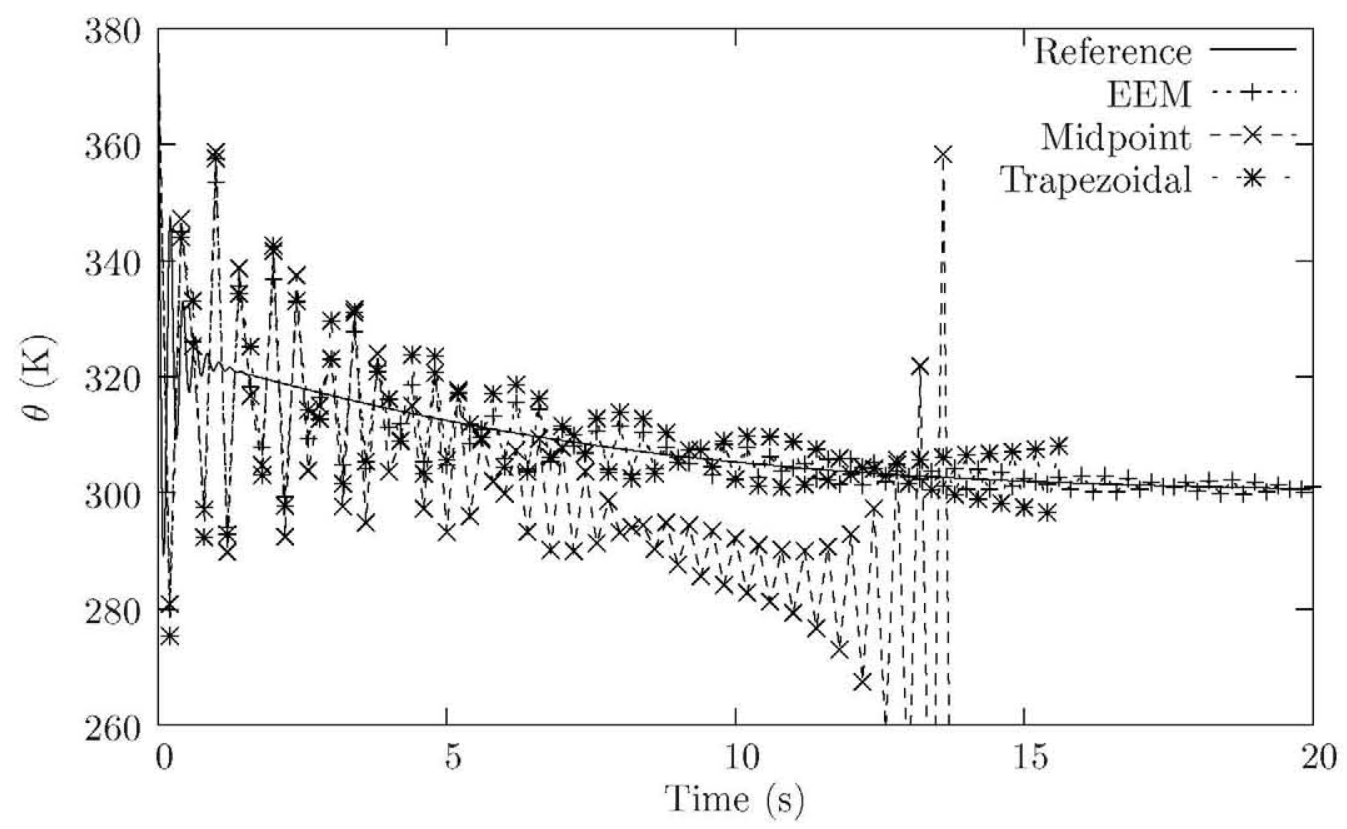

Figure 5: Temperature $\theta$ vs. time

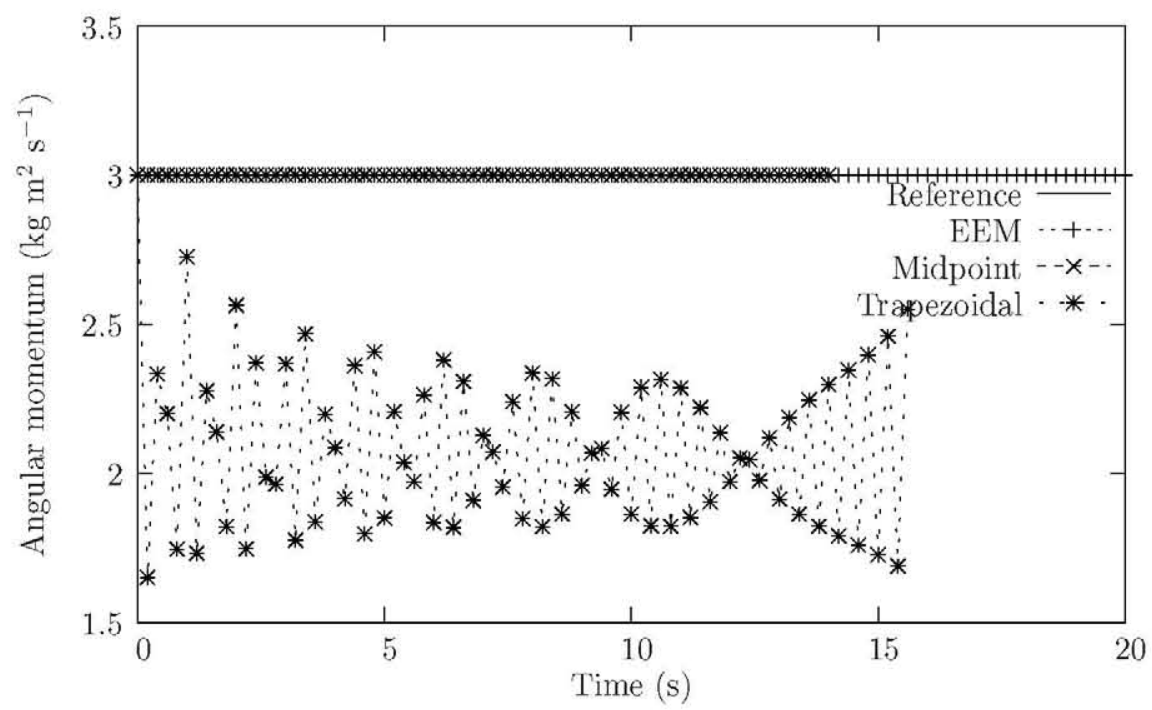

Figure 6: Angular momentum vs. time 


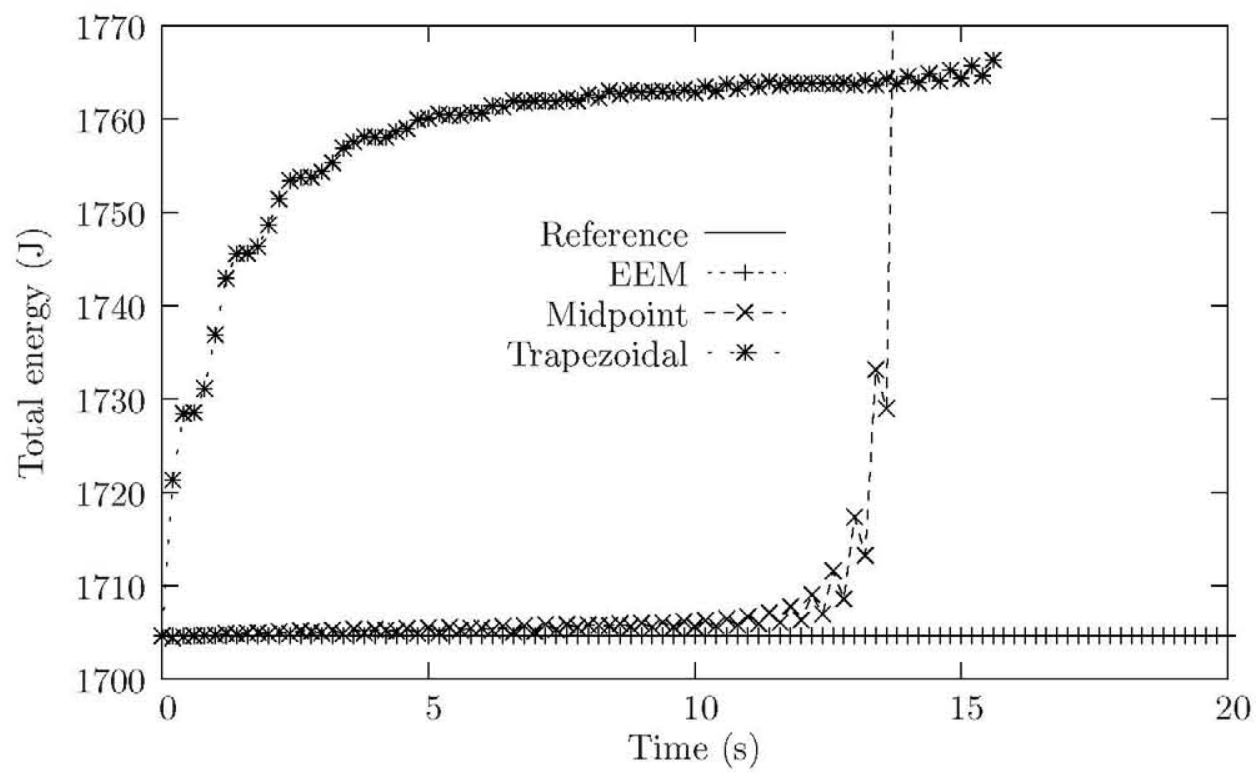

Figure 7: Total energy $E$ vs. time

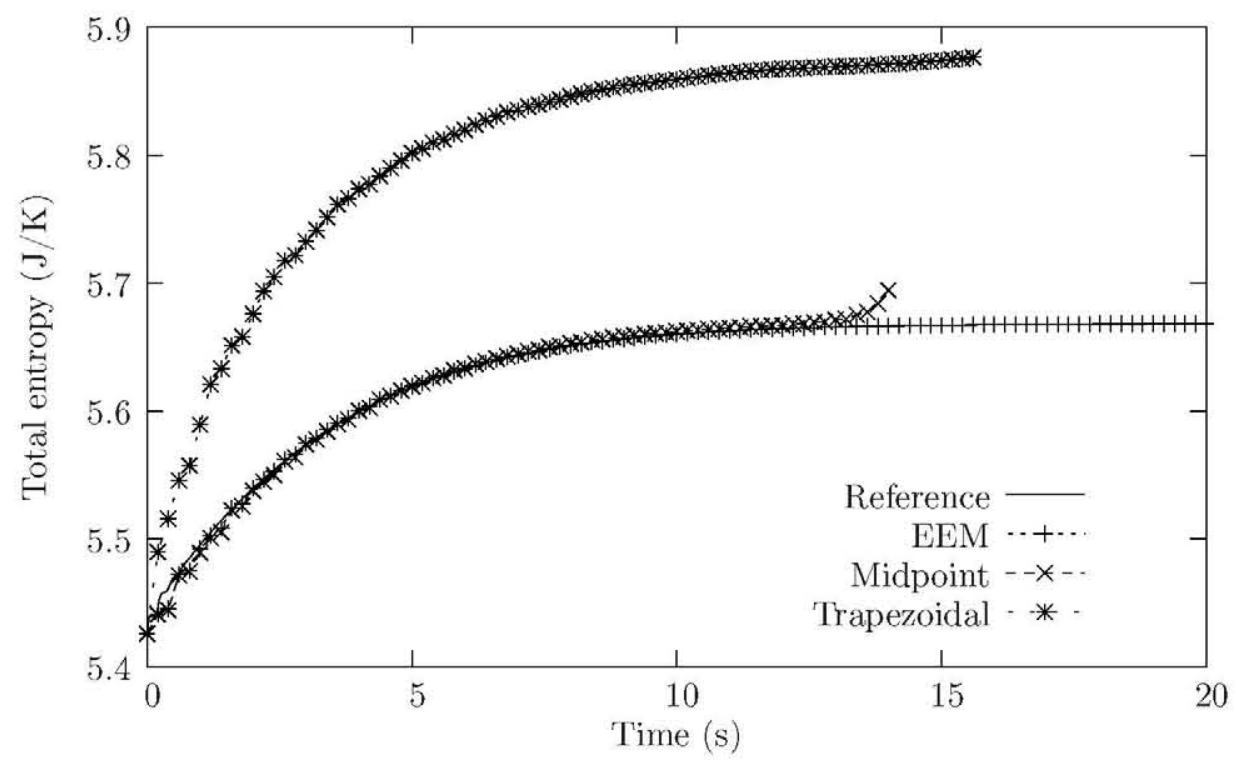

Figure 8: Total entropy $S$ vs. time 


\subsection{Satellite maneuver}

The second experiment simulates a maneuver of a satellite with flexible solar panels, with the model depicted in Figure 9. The body is a $1 \times 1 \times 1 \mathrm{~m}$ cube with a mass of $500 \mathrm{~kg}$, and each panel is $4 \mathrm{~m}$ long, $1 \mathrm{~m}$ wide and $0.1 \mathrm{~m}$ thick. Each panel is hinged to the satellite body by three smooth spherical joints and connected by four discrete thermo-visco-elastic elements, acting as stiffeners of the panels.

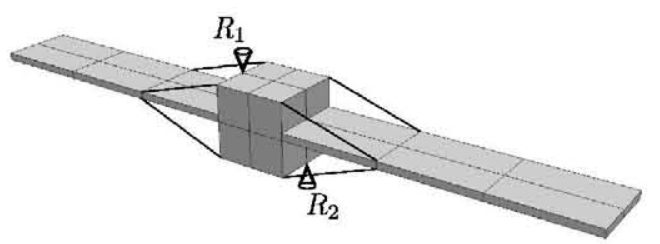

(a) Upper view, showing the rockets $R_{1}$ and $R_{2}$

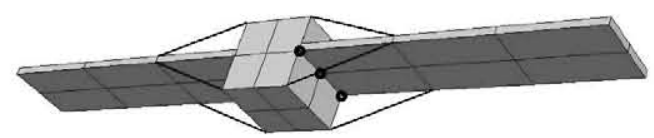

(b) Lower view, showing the hinges of the right solar panel with the satellite body.

Figure 9: Satellite model, showing the solar panels' hinges and the rockets

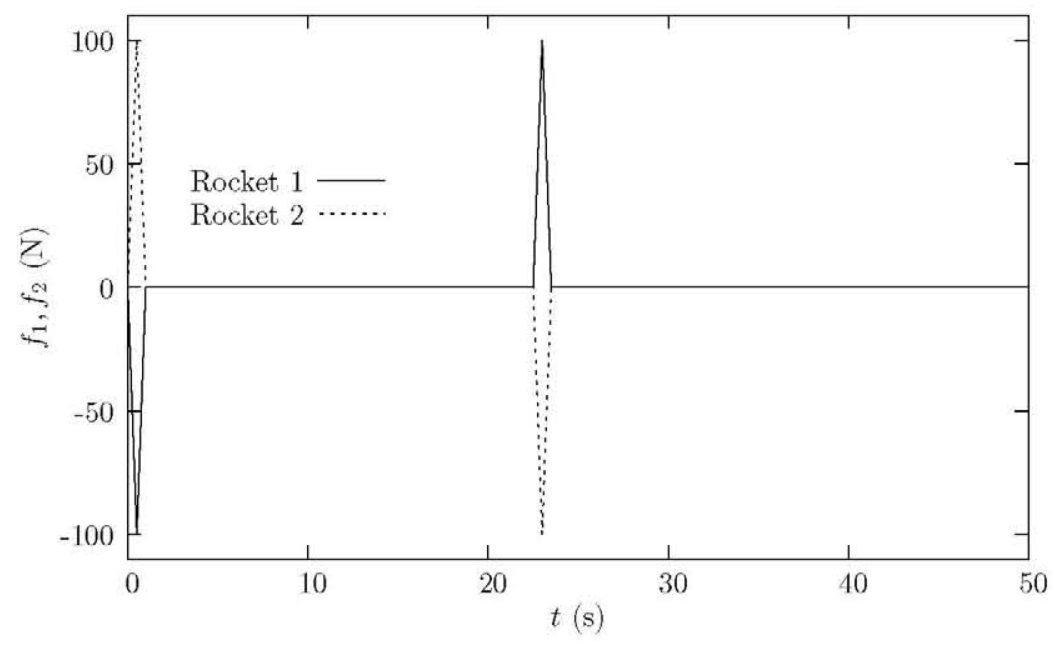

Figure 10: Rocket forces vs. time

The satellite body is equipped with two rockets $R_{1}$ and $R_{2}$ that provides the necessary thrust to perform the maneuver, aimed to change of attitude of the satellite in $90^{\circ}$. The maneuver starts firing both rockets during $1 \mathrm{~s}$, initiating a rotation of the whole satellite. After one and a half turn, the 
movement of the panels stabilizes and the rockets are fired again; this second firing takes place in the precise moment of time required to stop the rotation leaving the satellite with the new orientation. The time-dependent forces of the rockets are shown in Figure 10, and the motion is illustrated in Figure 11. 

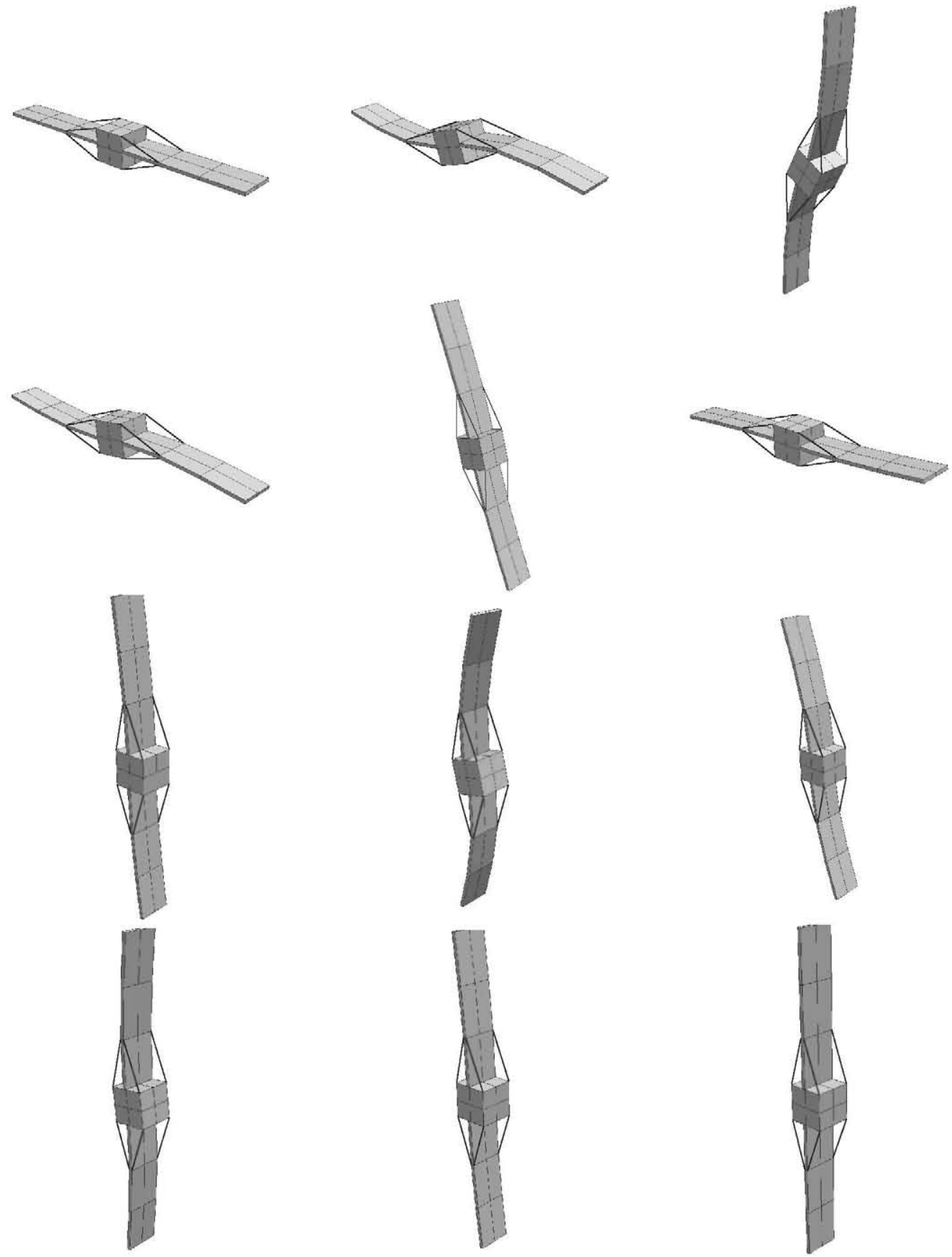

Figure 11: Snapshots of the maneuver (from top to bottom, left to right). Consistent integration, $\Delta t=0.2 \mathrm{~s}$ 
Both the satellite body and the panels are modeled with an homogeneous Saint Venant-Kirchhoff material. The material of the body has a density $\rho_{b}=500 \mathrm{~kg} / \mathrm{m}^{3}$, a Young modulus $E_{b}=1 \mathrm{GPa}$ and Poisson coefficient $\nu_{b}=0.3$. The panels have a density $\rho_{p}=10 \mathrm{~kg} / \mathrm{m}^{3}$, a Young modulus $E_{p}=5000 \mathrm{~Pa}$ and a Poisson coefficient $\nu_{p}=0.3$. Eight-node hexahedral finite elements with a fully nonlinear geometrical formulation are employed for both the body and the panels

The parameters of the thermo-visco-elastic stiffeners are $\lambda_{0}=1 \mathrm{~m}, \theta_{r}=$ $300 \mathrm{~K}, c_{10}=100 \mathrm{Nm}, c_{11}=0.5 \mathrm{NmK}^{-1}, \beta_{c}=4 \mathrm{NmK}^{-1}, c_{0}=1 \mathrm{NmK}^{-1}$, $\mu_{0}=5 \mathrm{Nm}^{-1}, \mu_{1}=0.1 \mathrm{Nm}^{-1} \mathrm{~K}^{-1}, \eta_{0}=100 \mathrm{Nsm}^{-1}, a=10 \mathrm{~K}$. The ambient temperature is $\theta_{r}=10 \mathrm{~K}$ and the thermal conductivity is $c=20 \mathrm{Nms}^{-1} \mathrm{~K}^{-1}$.

The motion obtained with the consistent integration of the dynamical equations is shown in Figure 11. The consistent formulation of the elastic parts of the system follows an energy-momentum scheme (Simó and Tarnow, 1992; Simó et al., 1992; González, 2000), and the dissipative thermo-viscoelastic stiffeners implements the energy-entropy-momentum formulation presented in this paper.

Figure 12 shows the evolution of the angular momentum, where the effect of the maneuvering rockets is clearly visible. Figure 13 shows the evolution of the energy for both the satellite and the ambient, and the total energy too. It is remarkable how the satellite looses energy, that is exactly transferred to the ambient maintaining the total energy constant when the rockets are not operative. Figure 14 shows the evolution of the entropy for both the satellite and the ambient, and the sum of both. It is remarkable how noticeable the effect of the rockets are, and how the total entropy never decreases. Figure 15 shows the evolution of the temperature of the eight stiffeners; again, the effect of the maneuvering rockets is clearly visible, and a symmetry between the stretched and compressed states is observed.

In order to analyze the behavior of the proposed method, a set of experiments are performed with different integrator schemes and different step sizes. The selected integrators are the implicit midpoint rule and the trapezoidal rule, with step sizes 0.1 and $0.2 \mathrm{~s}$. Figure 16 shows the evolution of the modulus of the angular momentum. Note that the midpoint rule apparently is stable and behaves correctly for $\Delta t=0.1 \mathrm{~s}$, computing a constant angular momentum during the free motion as expected. However, the trapezoidal rule does not have this property and exhibits a noticeable instability before the end of the simulation. For $\Delta t=0.2 \mathrm{~s}$, both the midpoint and the trapezoidal rule present clear instabilities in the first part of the simulation, before 


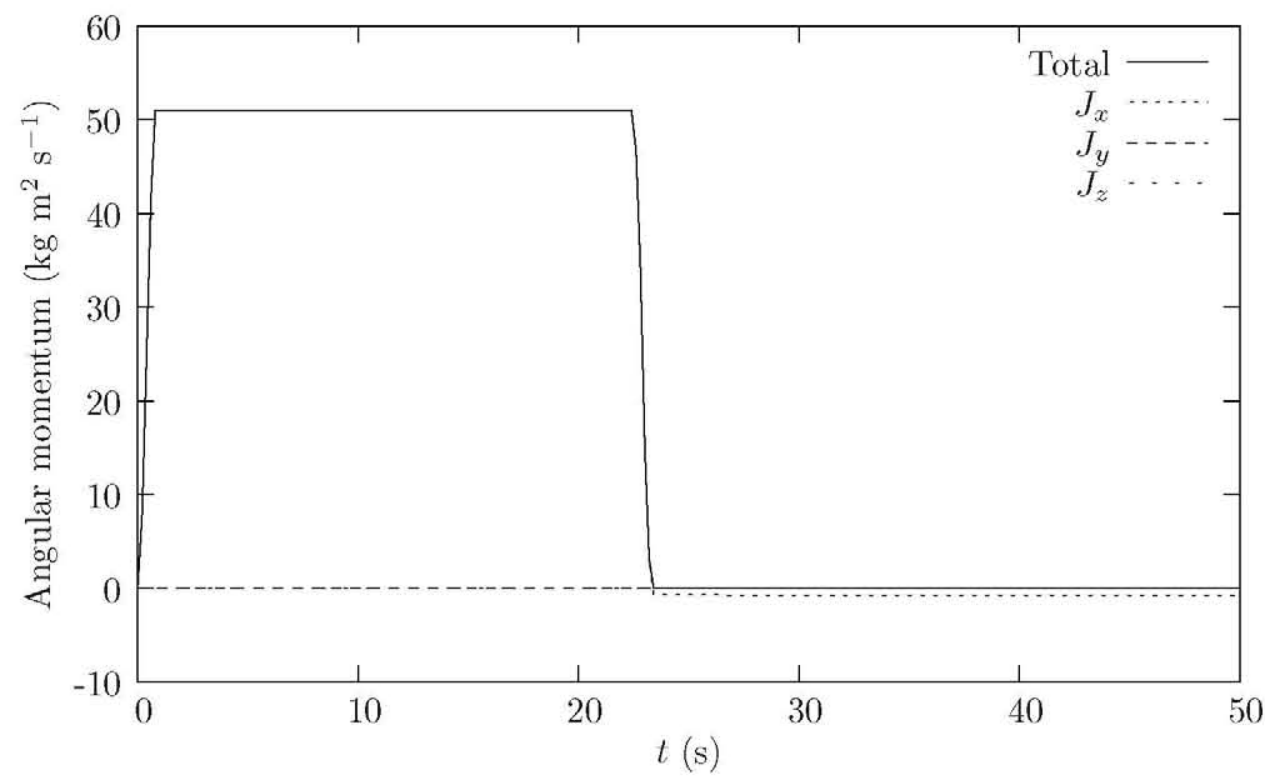

Figure 12: Angular momentum vs. time. Consistent integration, $\Delta t=0.2 \mathrm{~s}$

the second firing of the maneuvering rockets.

Figure 17 shows that the first principle of thermodynamics is violated by both the midpoint and the trapezoidal rule during the simulation; the effect is more apparent with the larger time step. The total entropy evolution is shown in Figure 18. Again, it should be noted that the midpoint behaves correctly for $\Delta t=0.1 \mathrm{~s}$, but it shows a large unrealistic increase in the first part of the simulation for $\Delta t=0.2 \mathrm{~s}$.

The behavior of the temperature in one of the stiffeners is shown in Figure 19. It is observed that the midpoint rule behaves correctly, apart from incipient instabilities that appear in the last part of the simulation for $\Delta t=0.1 \mathrm{~s}$. This instability shows up earlier as the time step increases, as shown in the plot for $\Delta t=0.2 \mathrm{~s}$. 


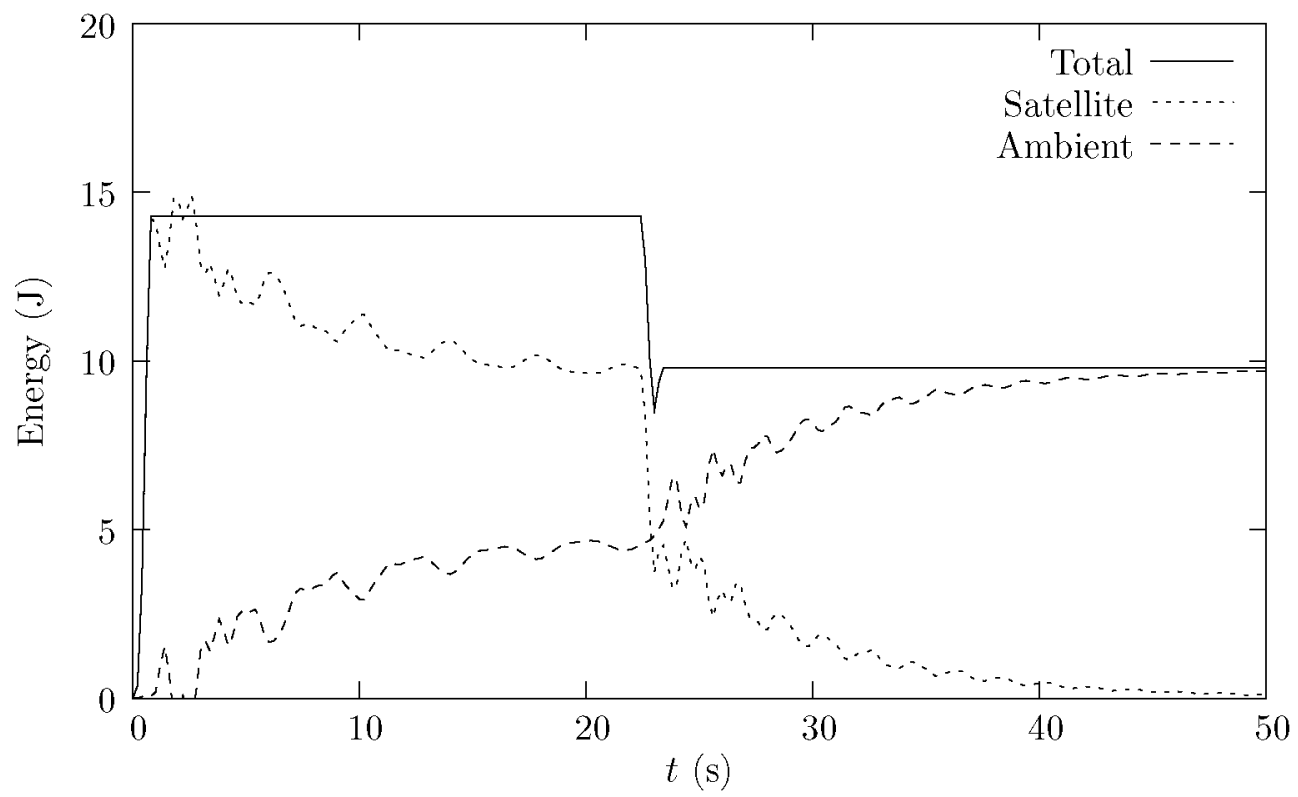

Figure 13: Energy vs. time. Consistent integration, $\Delta t=0.2 \mathrm{~s}$

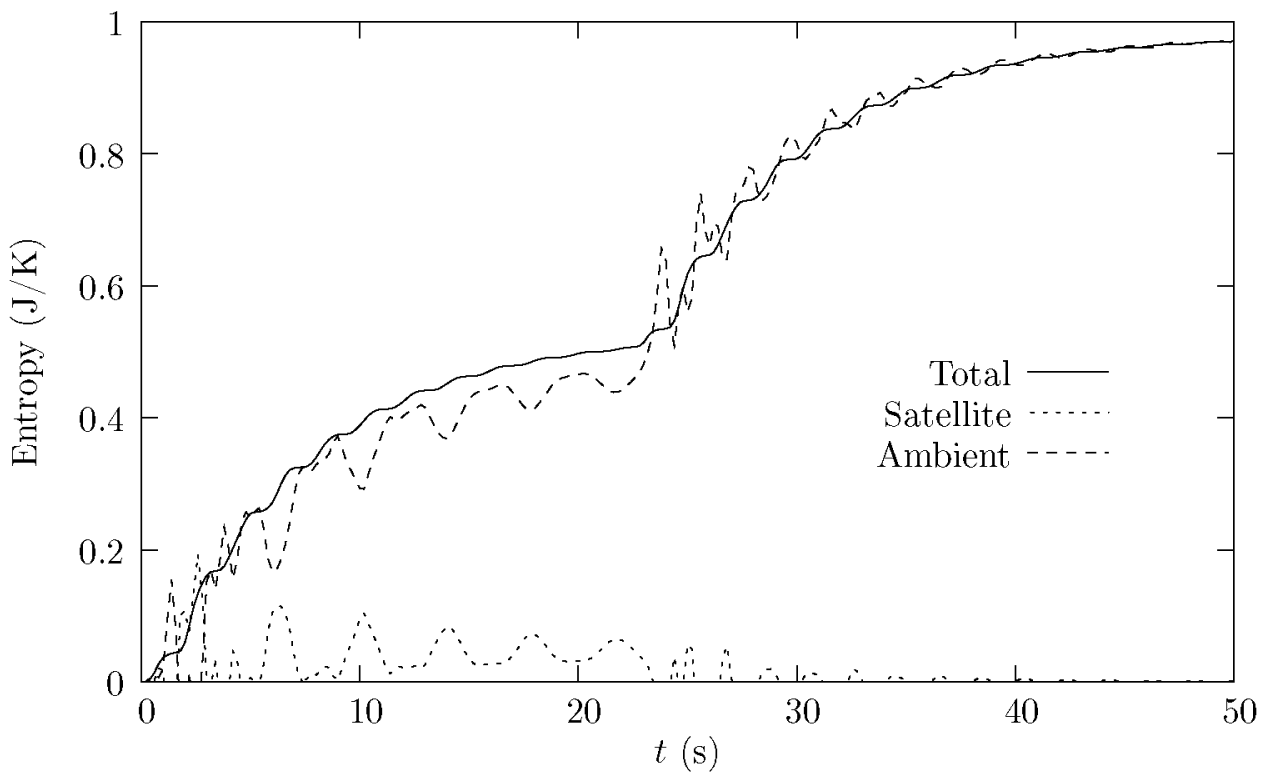

Figure 14: Entropy vs. time. Consistent integration, $\Delta t=0.2 \mathrm{~s}$ 


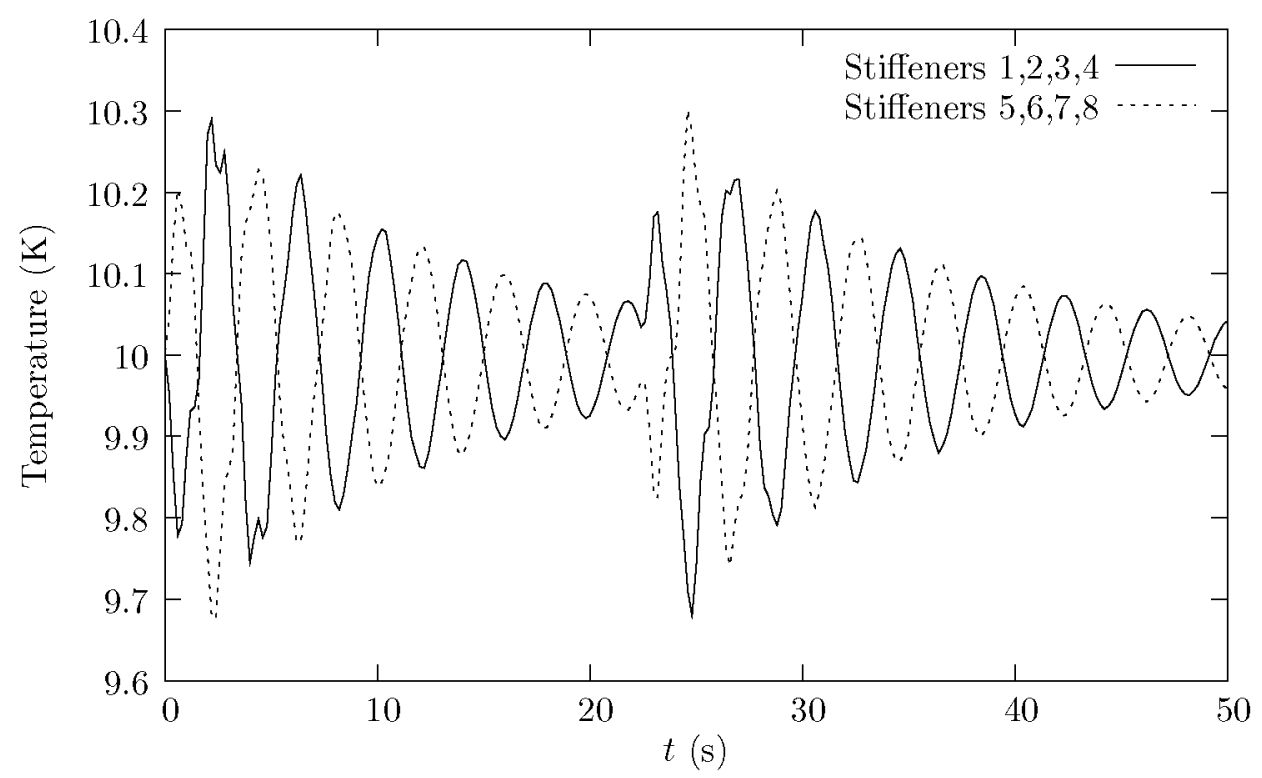

Figure 15: Stiffeners' temperature vs. time. Consistent integration, $\Delta t=0.2 \mathrm{~s}$ 


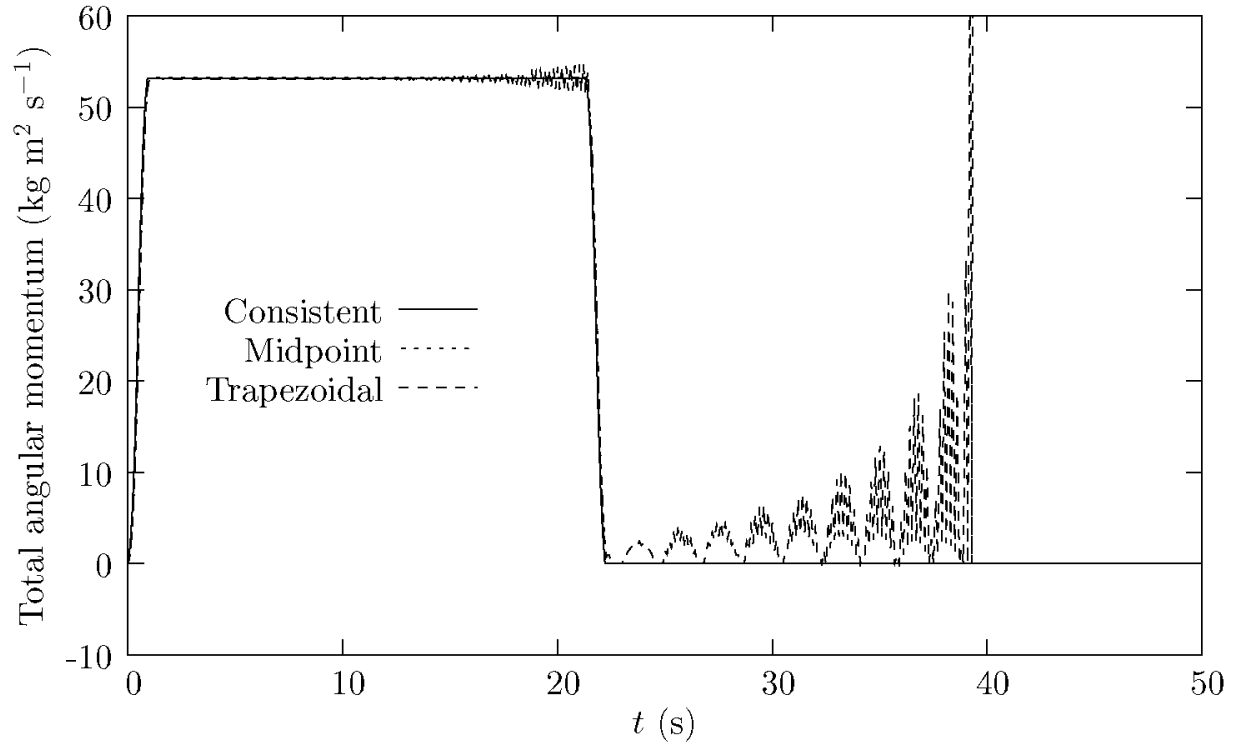

(a) $\Delta t=0.1$

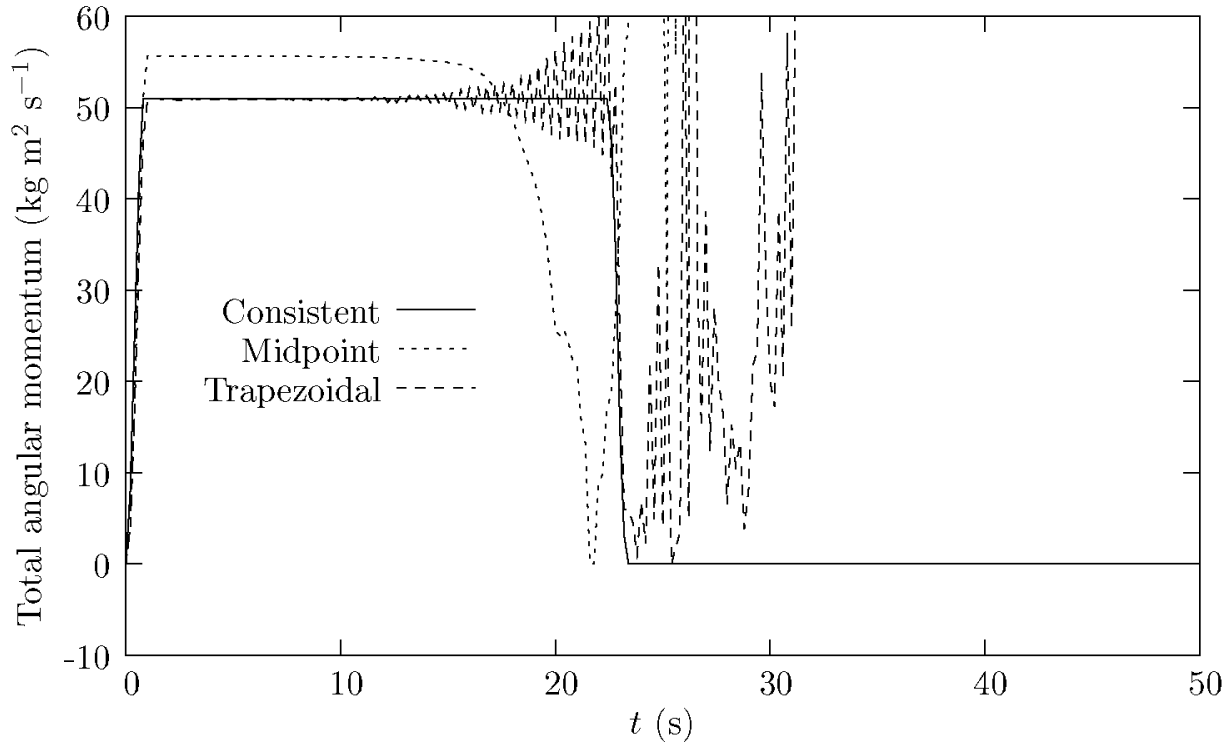

(b) $\Delta t=0.2 \mathrm{~s}$

Figure 16: Total angular momentum vs. time 


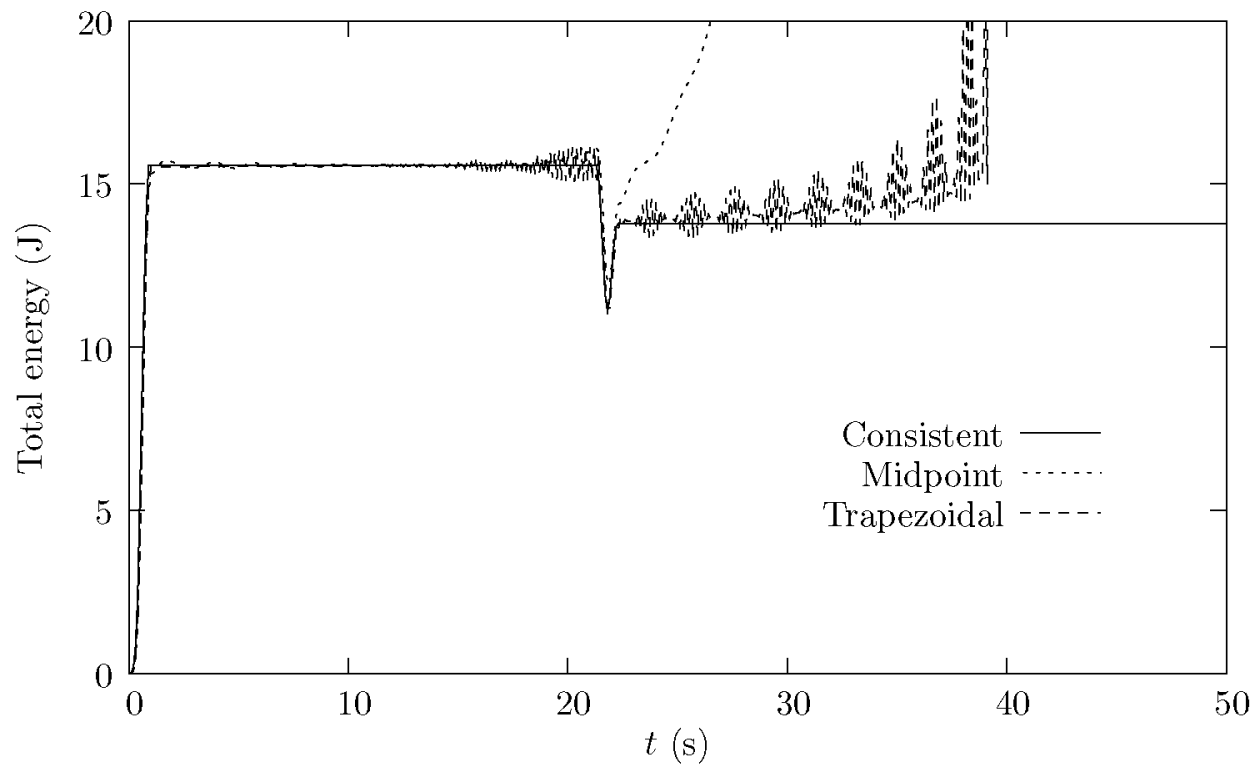

(a) $\Delta t=0.1 \mathrm{~s}$

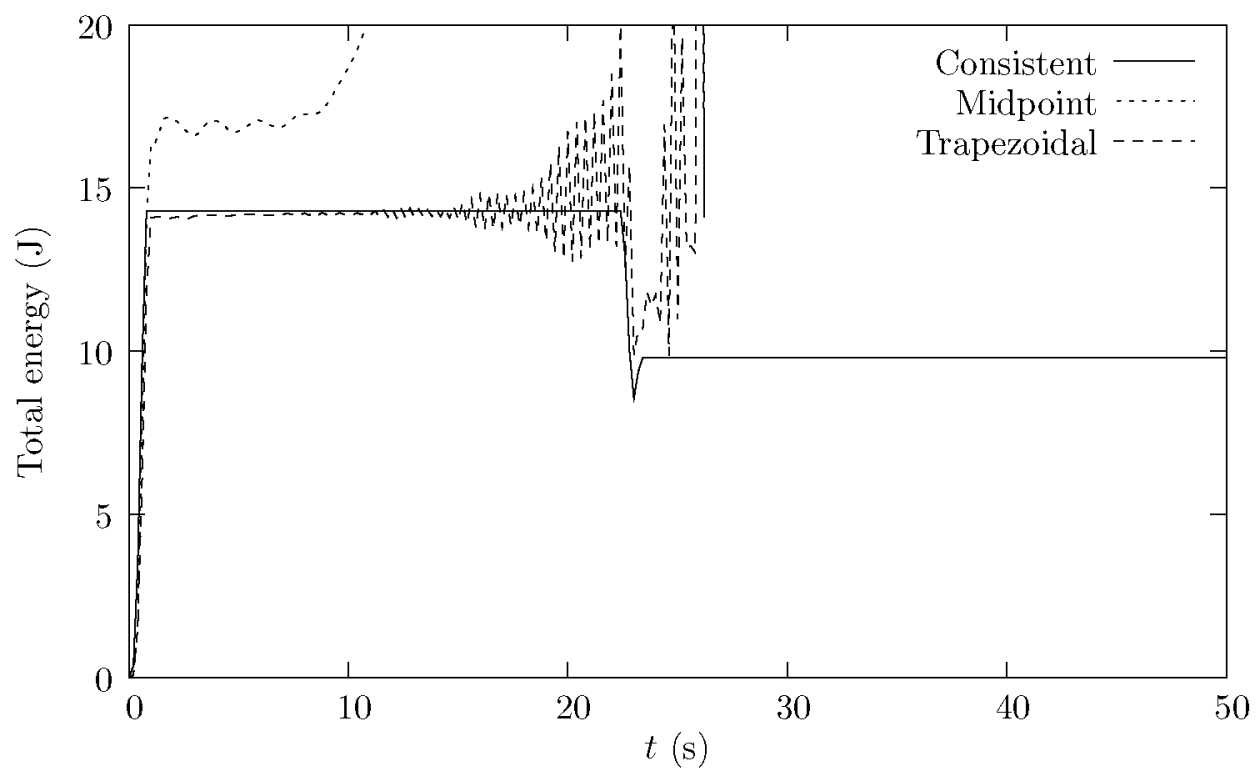

(b) $\Delta t=0.2 \mathrm{~s}$

Figure 17: Total energy (system + ambient) vs. time 


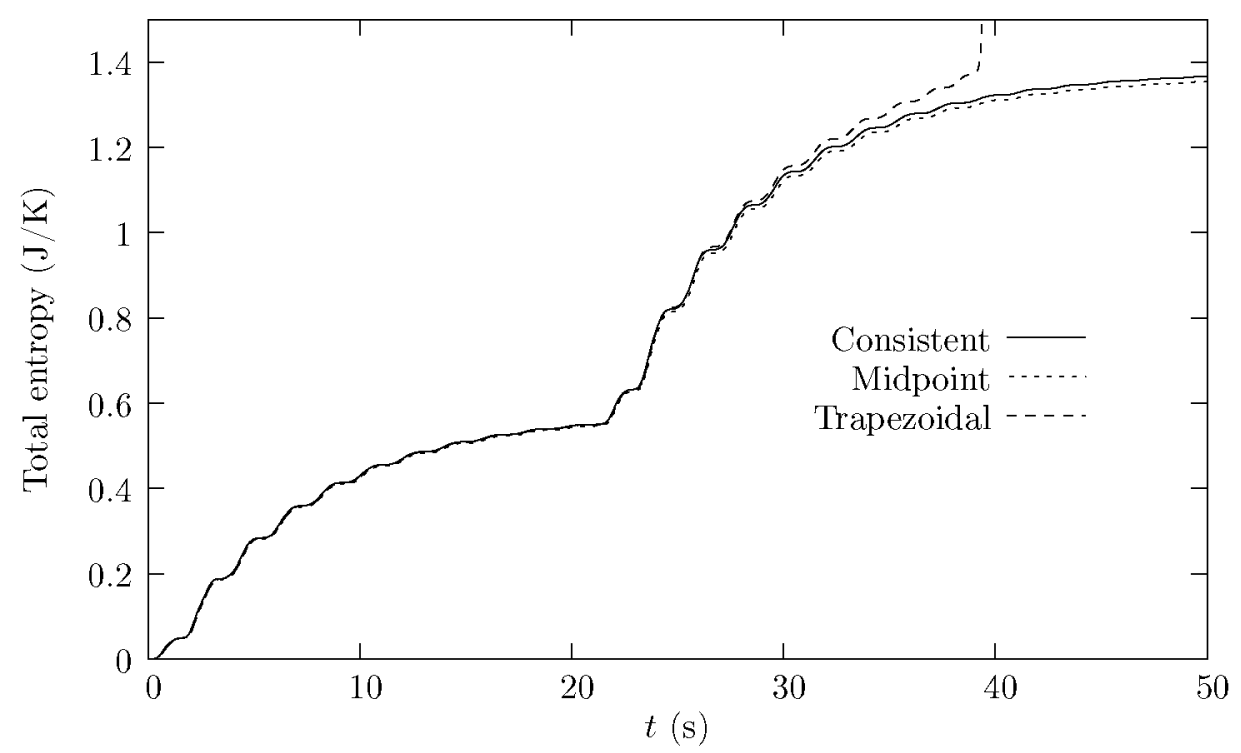

(a) $\Delta t=0.1 \mathrm{~s}$

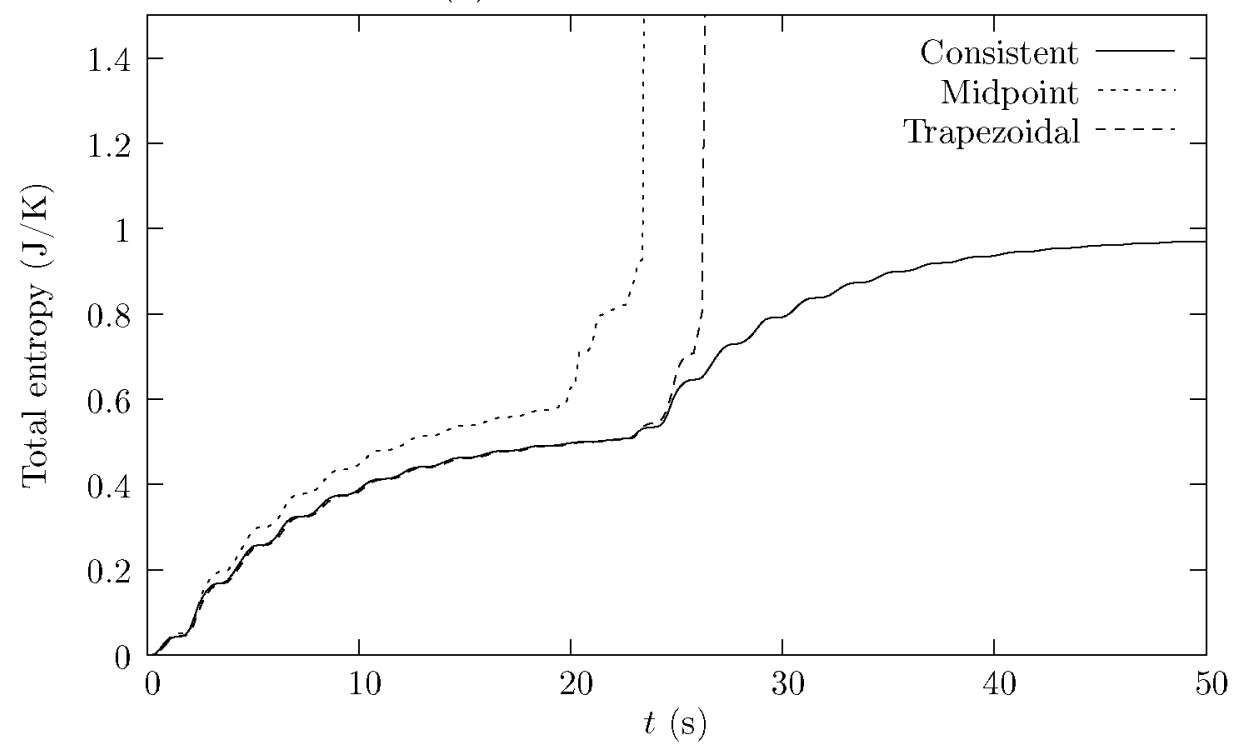

(b) $\Delta t=0.2 \mathrm{~s}$

Figure 18: Total entropy vs. time 


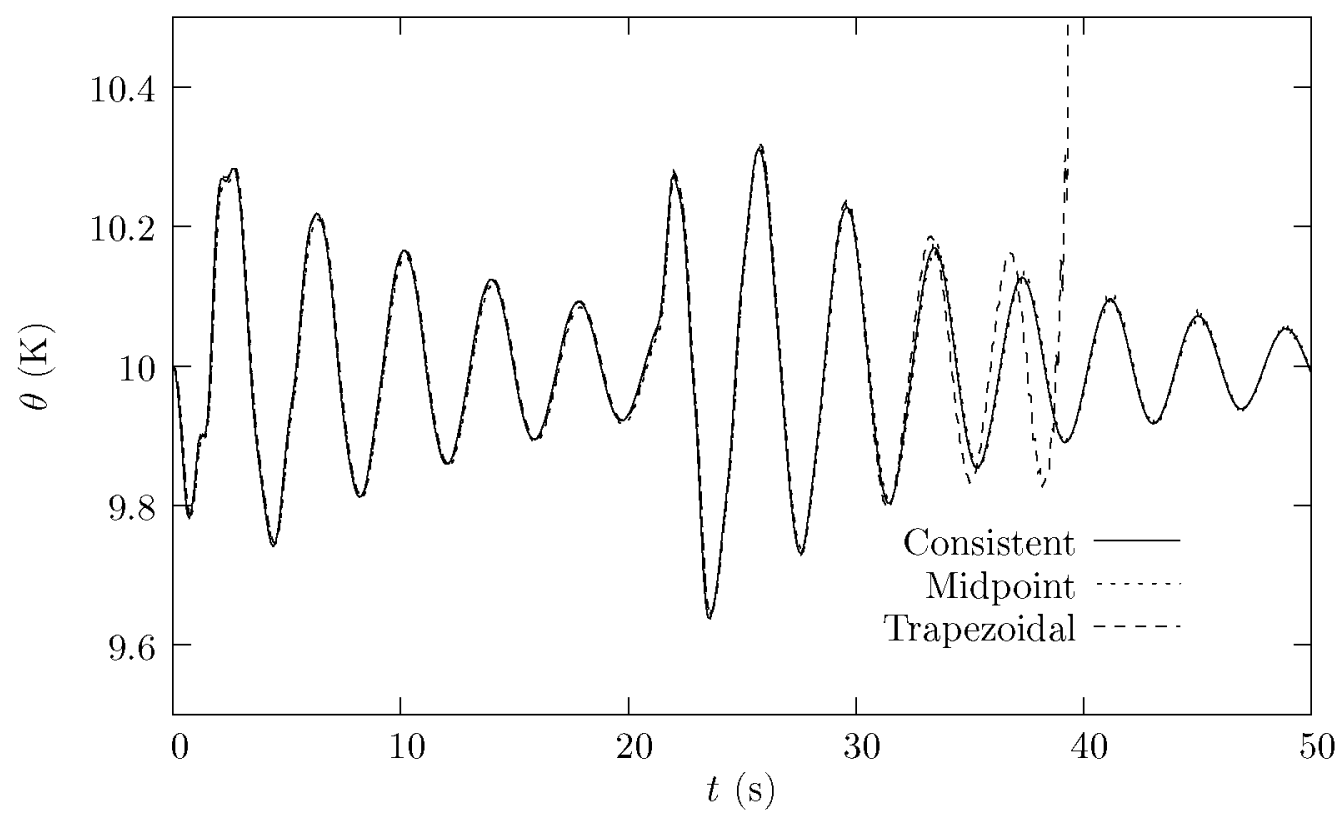

(a) $\Delta t=0.1 \mathrm{~s}$

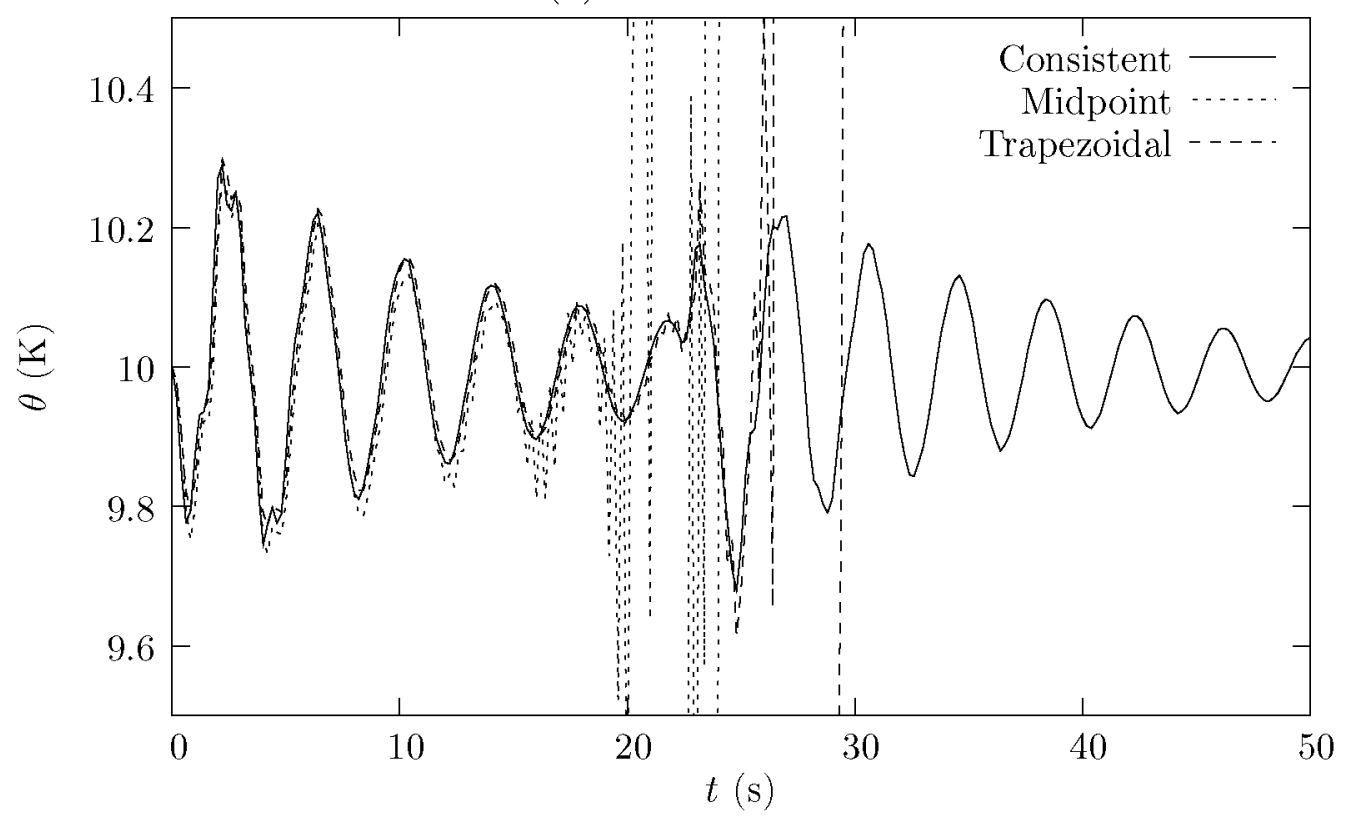

(b) $\Delta t=0.2 \mathrm{~s}$

Figure 19: Stiffener temperature vs. time 


\section{Conclusions}

We have presented in this paper a novel procedure to integrate the nonlinear dynamics of a discrete viscoelastic element with temperature effects. The element may experience large displacements, it may have a non linear elastic behavior, and incorporate stiffness and damping dependency on temperature. The element is immersed in a constant temperature environment, exchanging heat during the motion. To the authors' knowledge, this is the first fully thermodynamically consistent method for a thermomechanical problem that includes internal variables.

The proposed time-stepping procedure, which we refer to as the EnergyEntropy-Momentum method (EEM), is a second order accurate, implicit method that complies with both the first and second laws of thermodynamics in discrete form: the energy evaluated on the discrete motion remains constant, while the total entropy never decreases. The procedure also preserves the magnitudes related with the symmetries of the continuous evolution equations, namely the linear and angular momentum under free motion.

The numerical experiments suggest that the proposed method is more robust than comparable second order, implicit methods, namely the trapezoidal and midpoint rules. Moreover, the presented simulations indicate that the observed instabilities in these classical integrators are clearly related with their inability to control the energy growth of the solution and entropy. This type of observations is well documented in the literature of energy-momentum methods for elastodynamics, and we can speculate that in the current context, the loss of dissipative structure of the discrete evolution equations is responsible for the stability loss. In contrast with the trapezoidal and midpoint rules, the EEM method preserves the dissipative character of the solution, while preserving the laws of thermodynamics and the symmetries, as proved before. Moreover, the long term solutions obtained correspond to relative equilibria, suggesting that these trajectories are the attractors of the motion.

The method presented in this article is the finite-dimensional counterpart to a space-time discretization of finite strain thermo-visco-elasto-dynamics, a much more complex initial boundary value problem. An additional value of the proposed method is that it serves as guideline for the formulation of EEM algorithms for the infinite dimensional case, a problem on which we are currently working. 


\section{Acknowledgements}

Financial support for this work has been provided by the Spanish Ministry for Education and Science under grant DPI2009-14305-C02-02.

\section{References}

Armero, F., Romero, I., 2001a. On the formulation of high-frequency dissipative time-stepping algorithms for nonlinear dynamics. part i: low order methods for two model problems and nonlinear elastodynamics. Computer Methods in Applied Mechanics and Engineering 190 (20-21), 2603-2649.

Armero, F., Romero, I., 2001b. On the formulation of high-frequency dissipative time-stepping algorithms for nonlinear dynamics. part ii: secondorder methods. Computer Methods in Applied Mechanics and Engineering $190(51-52), 6783-6824$.

Armero, F., Romero, I., 2003. Energy-dissipative momentum-conserving time-stepping algorithms for the dynamics of nonlinear cosserat rods. Computational Mechanics 31, 3-26.

Bauchau, O. A., Bottasso, C. L., 1999. On the design of energy preserving and decaying schemes for flexible, nonlinear multi-body systems. Computer Methods in Applied Mechanics and Engineering 169 (1-2), 61-79.

Betsch, P., Uhlar, S., 2007. Energy-momentum conserving integration of multibody dynamics. Multibody System Dynamics 17, 243-289.

Borri, M., Bottasso, C. L., Trainelli, L., 2001. Integration of elastic multibody systems by invariant conserving/dissipating algorithms. i. formulation. Computer Methods in Applied Mechanics and Engineering 190 (2930), $3669-3699$.

Botasso, C. L., Borri, M., 1997. Energy preserving/decaying schemes for nonlinear beam dynamics using the helicoidal approximation. Comput. Meth. Appl. Mech. 143, 393-415.

Bottasso, C. L., Borri, M., Trainelli, L., 2001. Integration of elastic multibody systems by invariant conserving/dissipating algorithms. ii. numerical schemes and applications. Computer Methods in Applied Mechanics and Engineering 190 (29-30), $3701-3733$. 
García Orden, J. C., Goicolea, J. M., 2000. Conserving properties in constrained dynamics of flexible multibody systems. Multibody System Dynamics 4, 225-244.

García Orden, J. C., Goicolea, J. M., 2005. Advances in Computational Multibody Dynamics. Computational Methods in Applied Sciences. Springer-Verlag, Ch. Robust analysis of flexible multibody systems and joint clearances in an energy conserving framework, pp. 205-237.

Gear, C., 1971. Numerical Initial Value Problems in Ordinary Differential Equations. Prentice Hall.

González, O., 1996. Design and analysis of conserving integrators for nonlinear hamiltonian systems with symmetry. Ph.D. thesis, Stanford University Department of Mechanical Engineering.

González, O., 2000. Exact energy and momentum conserving algorithms for general models in nonlinear elasticity. Computer Methods in Applied Mechanics and Engineering 190, 1763-1783.

González, O., Simó, J., 1996. On the stability of symplectic and energymomentum algorithms for non linear hamiltonian systems with symmetry. Computer methods in Applied Mechanics and Engineering.

Groß, M., Betsch, P., 2010. Energy-momentum consistent finite element discretization of dynamic finite viscoelasticity. International Journal for $\mathrm{Nu}-$ merical Methods in Engineering 81, 1341-1386.

Groß, M., Betsch, P., Steinmann, P., 2005. Conservation properties of a time fe method. part iv: Higher order energy and momentum conserving schemes. International Journal for Numerical Methods in Engineering 63, $1849-1897$.

Hairer, E., Lubich, C., Wanner, G., 2002. Geometric numerical integration. Springer series in Computational Mathematics. Springer.

Holzapfel, G. A., Simó, J. C., 1996. A new viscoelastic constitutive model for continuous media at finite thermomechanical changes. International Journal of Solids and Structures 33 (20-22), 3019-3034. 
Kuhl, D., Crisfield, M., 1999. Energy-conserving and decaying algrithms in non-linear structuras dynamics. International Journal for Numerical Methods in Engineering 45, 569-599.

Kuhl, D., Ramm, E., 1996. Constraint energy momentum algorithm and its application to non-linear dynamics of shells. Computer Methods in Applied Mechanics and Engineering 136, 293-315.

Labudde, R. A., Greenspan, D., 1976a. Energy and momentum conserving methods of arbitrary order for the numerical integration of equations of motion - i. motion of a single particle. Numerische Mathematik 26, 323346.

Labudde, R. A., Greenspan, D., 1976b. Energy and momentum conserving methods of arbitrary order for the numerical integration of equations of motion - ii. motion of a system of particles. Numerische Mathematik 25, 323-346.

Leimkuhler, B., Reich, S., 2004. Simulating Hamiltonian dynamics. Cambridge monographs on applied and computational mathematics. Cambridge University Press.

Lion, A., Loose, S., 2002. A thermomechanically coupled model for automotive shock absorbers: theory, experiments and vehicle simulations on test tracks. Vehicle System Dynamics 37 (4), 241-261.

Meng, X. N., Laursen, T. A., 2002. Energy consistent algorithms for dynamic finite deformation plasticity. Computer Methods in Applied Mechanics and Engineering 191 (15-16), $1639-1675$.

Ortiz, M., Repetto, E., Stainier, L., 2000. A theory of subgrain dislocation structures in ductile single crystals. J. Mech. Phys. Solids 48 (10), 2077.

Öttinger, H., 2005. Beyond Equilibrium Thermodynamics. Wiley.

Richtmyer, R. D., Morton, K., 1967. Difference methods for initial value problems. John Wiley \& Sons, Inc., New York.

Romero, I., 2009. Thermodynamically consistent time-stepping algorithms for non-linear thermomechanical systems. International Journal for $\mathrm{Nu}$ merical Methods in Engineering 79, 706-732. 
Romero, I., 2010a. Algorithms for coupled problems that preserve symmetries and the laws of thermodynamics: Part i: Monolithic integrators and their application to finite strain thermoelasticity. Computer Methods in Applied Mechanics and Engineering 199 (25-28), 1841-1858.

Romero, I., 2010b. Algorithms for coupled problems that preserve symmetries and the laws of thermodynamics. part ii: Fractional step methods. Computer Methods in Applied Mechanics and Engineering DOI: 10.1016/j.cma.2010.03.016.

Romero, I., Armero, F., 2000. High frequency dissipative time-stepping algorithms for the dynamics of nonlinear shells. In: European Congress on Computational Methods in Applied Sciences and Engineering, ECCOMAS 2000. Barcelona.

Sanz-Serna, J. M., Calvo, M., 1994. Numerical Hamiltonian problems. Chapman and Hall.

Simó, J., Tarnow, N., 1992. The discrete energy-momentum method. conserving algorithms for nonlinear elastodynamics. Zeitschrift für Angewandte Mathematik und Physik (ZAMP) 43 (5), 757-792.

Simó, J., Tarnow, N., Wong, K., 1992. Exact energy-momentum conserving algorithms and symplectic schemes for non-linear dynamics. Computer methods in Applied Mechanics and Engineering 100, 63-116.

Simo, J. C., Tarnow, N., 1994. A new energy and momentum conserving algorithm for the non-linear dynamics of shells. Int. J. Numer. Meth. Eng. $37(15), 2527-2549$.

Tarnow, N., Simo, J. C., 1994. How to render second order accurate timestepping algorithms fourth order accurate while retaining the stability and conservation properties. Comput. Meth. Appl. Mech. 115 (3-4), 233-252. 